\title{
Differentially Private Significance Tests for Regression Coefficients
}

\author{
Andrés F. Barrientos, Jerome P. Reiter, Ashwin Machanavajjhala, Yan Chen *
}

\begin{abstract}
Many data producers seek to provide users access to confidential data without unduly compromising data subjects' privacy and confidentiality. One general strategy is to require users to do analyses without seeing the confidential data; for example, analysts only get access to synthetic data or query systems that provide disclosure-protected outputs of statistical models. With synthetic data or redacted outputs, the analyst never really knows how much to trust the resulting findings. In particular, if the user did the same analysis on the confidential data, would regression coefficients of interest be statistically significant or not? We present algorithms for assessing this question that satisfy differential privacy. We describe conditions under which the algorithms should give accurate answers about statistical significance. We illustrate the properties of the proposed methods using artificial and genuine data.
\end{abstract}

Keywords: Confidentiality, Disclosure, Laplace, Query, Synthetic, Verification

*Andrés F. Barrientos is Postdoctoral Associate, Department of Statistical Science, Duke University, Durham, NC 27708 (email: afb26@ stat.duke.edu); Jerome Reiter is Professor, Department of Statistical Science, Duke University, Durham, NC 27708 (jerry@stat.duke.edu); Ashwin Machanavajjhala is Assistant Professor, Department of Computer Science, Duke University, Durham, NC 27708 (ashwin@cs.duke.edu); and, Yan Chen is Graduate Student, Department of Computer Science, Duke University, Durham, NC 27708 (yanchen@cs.duke.edu). 


\section{Introduction}

In many settings, data producers such as national statistical agencies, survey organizations, health systems, and private sector companies — henceforth all called agencies-seek to provide researchers and the broader public access to data on individual records. However, these agencies are ethically and often legally obligated to protect the confidentiality of data subjects' identities and sensitive attributes. Research has shown that stripping obvious identifiers, like names and addresses, may not suffice to protect confidentiality (e.g., Sweeney, 1997, 2013; Narayanan and Shmatikov, 2008; Parry and Chase, 2011). Ill-intentioned users-henceforth called intrudersmay be able to learn sensitive information by linking released data files to records in external databases by matching on fields common to both datasets, thereby breaking the protection from de-identification.

In recognition of this threat, agencies have developed and deployed techniques that allow users to do analyses without seeing the actual data. One approach is to use remote access query systems (Gomatam et al., 2005) in which the user submits a query to a server that holds the data for output from some statistical model. The server runs the query and reports back the analysis results to the user, e.g., estimated regression coefficients and their standard errors, without ever allowing the user to see the individual-level data. To further reduce disclosure risks, the outputs usually are coarsened or perturbed (O'Keefe and Chipperfield, 2013); for example, the server can add noise to outputs that satisfies the risk criterion differential privacy (Dwork et al., 2006; Wasserman and Zhou, 2010). Query system approaches are used by many government agencies, such as the Census Bureau and the Australian Bureau of Statistics, and are being implemented for general social science data access, for example in DataVerse (King, 2007; Crosas, 2011) and in the Private data Sharing Interface (Gaboardi et al. 2016a). A second approach is to release fully synthetic data (Rubin, 1993; Raghunathan et al., 2003; Reiter, 2005; Drechsler, 2011). Here, the agency generates new values for every confidential datum by sampling from a predictive distribution estimated with the confidential data. Since all values are simulated, it is 
nonsensical for intruders to match released cases to external records. Synthetic data have been used in several public use data products, including the Longitudinal Business Database Kinney et al., 2011), the Survey of Income and Program Participation (Abowd et al., 2006), and the OnTheMap application (Machanavajjhala et al., 2008).

While query systems and synthetic data are appealing options for data release, they have a significant drawback: it is difficult for analysts to know how much they should trust the results of their analyses. For example, in a query system, the user might ask for outputs from a regression model that, in actuality, fits poorly on the confidential data. This lack of fit cannot be easily detected from the coefficients and standard errors alone, whether they are perturbed or not. Additionally, the steps taken to perturb the outputs could infuse substantial error into the reported coefficients. Similar dilemmas arise for synthetic data. By default, the synthetic data reflect only those distributional features and relationships encoded in the synthesis models (Reiter, 2005). The synthesis models may fail to describe the data in ways that lead the analyst to findings that are not supported by the confidential data. Further, even when the synthesis models adequately describe the distributions in the confidential data, the process of generating synthetic data tends to increase standard errors, which could obscure important relationships.

The literature on privacy-preserving data analysis has begun to address aspects of this problem. Reiter (2003) suggests that linear regression output from query systems be accompanied by synthetic plots of residuals versus predicted values. Related residual diagnostics for logistic regressions are proposed in Reiter and Kohnen (2005) and O'Keefe and Good (2009). Chen et al. (2016) present an algorithm for releasing residual plots (and also an algorithm for ROC curves for logistic regression) that satisfies differential privacy. Their algorithm takes as input the privately-estimated coefficients, which could come from noisy outputs or a synthetic data analysis. While useful diagnostic tools, these plots do not provide analysts with means to compare inferences obtained via the privacy-preserving mechanism to those that would be obtained from the confidential data. It may be, for example, that a particular regression coefficient of substantive interest has a large p-value in the noisy output or synthetic data, even though it has 
a small one in the confidential data, or vice versa.

In this article, we present algorithms for comparing the sign and significance level of privatelycomputed regression coefficients, i.e., those computed via output perturbation or via synthetic data, with those computed from the confidential data. We envision the outputs of these algorithms being delivered to users via a verification server (Reiter et al., 2009). This is a query system that allows users to ask for measures indicating how similar privately-computed results are to those based on the confidential data without allowing users to see the confidential data. The algorithms satisfy differential privacy, which has important benefits in this interactive context. As shown in Reiter et al. (2009) and McClure and Reiter (2012), when verification servers provide exact (unperturbed) answers to queries about similarity of results, intruders can query the server repeatedly to gather information that, in combination, provides unacceptably tight ranges for individual confidential values. Differential privacy provides provable bounds for the amount of information leaked by the server over repeated queries, regardless of their nature.

The basic idea of the algorithms is built on the subsample and aggregate mechanism of Nissim et al. (2007). We randomly partition the confidential data into $M$ disjoint subsets. In each subset, we estimate the regression using only the data in that subset, from which we compute the univariate $t$-statistic for the regression coefficient(s) of interest to the user. We truncate each $t$-statistic at some user-defined threshold $a$; this facilitates differentially private algorithm design, as we discuss later. We add noise to the average of the truncated $t$-statistics, sampled from a Laplace distribution with variance tuned to satisfy differential privacy. We refer the resulting noisy statistic to an appropriate reference distribution under the null hypothesis that the coefficient equals zero, resulting in calibrated p-values. The p-value can be used directly as evidence of the significance of the coefficient, or it can be compared with the corresponding, privatelycomputed $\mathrm{p}$-value for purposes of verification. The sign of the noisy $t$-statistic also provides a differentially private estimate of the sign of the coefficient.

We are not aware of algorithms for differentially private significance tests for linear regression coefficients, although the literature on differential privacy includes significance tests for 
models appropriate for other settings. Several authors have developed differentially private significance tests for categorical data and contingency tables (e.g., Vu and Slavkovic, 2009; Gaboardi et al., 2016b; Wang et al., 2017). These tests cannot be sensibly used for linear regression, where the outcome variable, as well as potentially some of the explanatory variables, are assumed to be continuous rather than categorical. Solea (2014) and D'Orazio et al. (2015) propose differentially private significance tests for the mean and the difference of means of Gaussian random variables, respectively. These authors assume that bounds for the means or the data values are known, whereas we work in multivariate regression settings where bounds on the variables need not be known. Campbell et al. (2018) propose a differentially private algorithm for analysis of variance, which is a special case of linear regression with only categorical explanatory variables. They restrict results to outcomes that lie on the unit interval, do not consider continuous predictors, and report only the result of the omnibus significance test that all coefficients simultaneously equal zero. Karwa and Vadhan (2017, 2018) propose differentially private algorithms to obtain confidence intervals for single means of Gaussian random variables, which can be inverted to significance tests. Their approach relies on spending some privacy budget to bound the range and standard deviation of the data values for the single variable. Their approach does not apply in a straightforward manner to regression modeling with multiple explanatory variables, as the expression for the variance of any estimated regression coefficient has a numerator and denominator that are non-linear functions of all the variables used in the regression model.

Multiple authors have developed differentially private algorithms for estimating pieces of the outputs needed for significance testing of coefficients in linear regressions or other predictive models (e.g., Sarlós, 2006; Chaudhuri and Monteleoni, 2009; Dwork and Lei, 2009; Chaudhuri et al., 2011; Kifer et al., 2012; Zhang et al., 2012; Bassily et al., 2014; Karwa et al., 2015; Wu et al., 2015; Honkela et al., 2016; Jing et al., 2018). None of these works includes procedures to compute standard errors, making it impossible to conduct significance tests. Sheffet (2015) presents an algorithm for estimating regressions that does provide standard errors and hence 
significance tests; however, the algorithm sometimes returns output associated with a variant of ridge regression rather than strictly linear regression. The algorithm also requires all data values to be bounded, which we do not require in our algorithms. Finally, the algorithm appears to require $(\epsilon, \delta>0)$-differential privacy to give useful outputs, whereas our algorithm allows for $\epsilon$-differential privacy,

The remainder of the article is organized as follows. In Section 2, we review differential privacy and some of the techniques used to design algorithms that satisfy it. In Section 3 , we present the algorithm for the differentially private $t$-statistic, including its reference distribution. In Section 4 , we discuss some theoretical aspects of the differentially private $t$-statistic, focusing on approximation properties and type II error rates. In Section 5, we present results of simulation studies that illustrate the performance of the differentially private $t$-statistic in finite samples. In Section 6, we present an approach for choosing the number of partitions and the threshold level. These drive the accuracy and usefulness of the inferences. In Section 7 we conclude with suggestions for implementation of these techniques, as well as discuss future research topics around verification of privately-computed regression quantities.

\section{Review of Differential Privacy}

Before reviewing differential privacy, we motivate why one should not simply release verification measures without redaction. Suppose that an intruder asks a verification server to return the value of the $t$-statistic for the slope in a regression of an outcome $y$ on a single predictor $x$, and that the verification server provides this value from the regression estimated with the confidential data. Consider a worst case scenario: the intruder knows the values of $\left(x_{i}, y_{i}\right)$ for all but one record in the confidential data. If the intruder submits a regression involving all records and then requests the $t$-statistic, the user can try various combinations of $(x, y)$ for that unknown record until finding the set of values that yield the reported $t$-statistic. More generally, similar attacks work for an intruder who knows the values of $\left(x_{i}, y_{i}\right)$ for any $r$ records in the 
confidential data when the intruder can request output from a regression estimated with those $r$ records plus one additional record.

As these examples illustrate, it is desirable to redact the verification measures before releasing them, which we do using differential privacy. Let $\mathcal{A}$ be an algorithm that takes as input a database $\mathbf{D}$ and outputs some quantity $o$, i.e., $\mathcal{A}(\mathbf{D})=o$. In our context, these outputs are used to form verification measures for the $t$-statistic and sign. Define neighboring databases, $\mathbf{D}$ and $\mathbf{D}^{\prime}$, as databases that differ in one row and are identical for all other rows. Specifically, $\mathbf{D}$ and $\mathbf{D}^{\prime}$ are neighboring databases if there exists only one record $d \in \mathbf{D}$ and one record $d^{\prime} \in \mathbf{D}^{\prime}$ such that $d \neq d^{\prime}$ and $\mathbf{D}-\{d\}=\mathbf{D}^{\prime}-\left\{d^{\prime}\right\}$.

Definition 1 ( $\epsilon$-differential privacy). An algorithm $\mathcal{A}$ satisfies $\epsilon$-differential privacy if for any pair of neighboring databases $\left(\mathbf{D}, \mathbf{D}^{\prime}\right)$, and any non-negligible measurable set $S \subseteq$ range $(\mathcal{A})$, the $\operatorname{Pr}(\mathcal{A}(\mathbf{D}) \in S) \leq \exp (\epsilon) \operatorname{Pr}\left(\mathcal{A}\left(\mathbf{D}^{\prime}\right) \in S\right)$.

Intuitively, $\mathcal{A}$ satisfies $\epsilon$-DP when the distributions of its outputs are similar for any two neighboring databases, where similarity is defined by the factor $\exp (\epsilon)$. The $\epsilon$, also known as the privacy budget, controls the degree of the privacy offered by $\mathcal{A}$, with lower values implying greater privacy guarantees. $\epsilon$-DP is a strong criterion, since even an intruder who has access to all of $\mathrm{D}$ except any one row learns little from $\mathcal{A}(\mathrm{D})$ about the values in that unknown row when $\epsilon$ is small.

Differential privacy has three other properties that are appealing for verification measures. Let $\mathcal{A}_{1}(\cdot)$ and $\mathcal{A}_{2}(\cdot)$ be $\epsilon_{1}$-DP and $\epsilon_{2}$-DP algorithms. First, for any database $\mathbf{D}$, releasing the outputs of both $\mathcal{A}_{1}(\mathbf{D})$ and $\mathcal{A}_{2}(\mathbf{D})$ ensures $\left(\epsilon_{1}+\epsilon_{2}\right)$-DP. Thus, we can quantify and track the total privacy leakage from releasing verification measures. Second, releasing the outputs of both $\mathcal{A}_{1}\left(\mathbf{D}_{1}\right)$ and $\mathcal{A}_{2}\left(\mathbf{D}_{2}\right)$, where $\mathbf{D}_{1} \cap \mathbf{D}_{2}=\emptyset$, satisfies $\max \left\{\epsilon_{1}, \epsilon_{2}\right\}$-DP. Third, for any algorithm $\mathcal{A}_{3}(\cdot)$, releasing $\mathcal{A}_{3}\left(\mathcal{A}_{1}(\mathbf{D})\right)$ for any $\mathbf{D}$ still ensures $\epsilon_{1}$-DP. Thus, post-processing the output of $\epsilon$-DP algorithms does not incur extra loss of privacy.

A common method for ensuring $\epsilon$-DP is the Laplace Mechanism (Dwork et al., 2006). For 
any function $f: \mathbf{D} \rightarrow \mathbb{R}^{d}$, let $\Delta(f)=\max _{\left(\mathbf{D}_{1}, \mathbf{D}_{2}\right)}\left\|f\left(\mathbf{D}_{1}\right)-f\left(\mathbf{D}_{2}\right)\right\|_{1}$, where $\left(\mathbf{D}_{1}, \mathbf{D}_{2}\right)$ are neighboring databases. This quantity, known as the global sensitivity of $f$, is the maximum $L_{1}$ distance of the outputs of the function $f$ between any two neighboring databases. The Laplace Mechanism is

$$
\mathbf{L M}(\mathbf{D})=f(\mathbf{D})+\eta,
$$

where $\eta$ is a $d \times 1$ vector of independent draws from a Laplace distribution with density $p(x \mid$ $\lambda)=(1 /(2 \lambda)) \exp (-|x| / \lambda)$, where $\lambda=\Delta(f) / \epsilon$. We use the Laplace Mechanism to design verification measures that satisfy $\epsilon$-differential privacy, which we refer to as $\epsilon$-DP verification measures.

We also use the subsample and aggregate technique (Nissim et al., 2007). This technique allows us to reduce the global sensitivity of $f$, thereby reducing the variance in the noise distribution. To implement this technique, we randomly partition the dataset $\boldsymbol{D}$ into $M$ disjoint subsets, $\boldsymbol{D}_{1}, \ldots, \boldsymbol{D}_{M}$. We then compute $f\left(\boldsymbol{D}_{1}\right), \ldots, f\left(\boldsymbol{D}_{M}\right)$ and their average $M^{-1} \sum_{l=1}^{M} f\left(\boldsymbol{D}_{l}\right)$. The

global sensitivity of $M^{-1} \sum_{l=1}^{M} f\left(\boldsymbol{D}_{l}\right)$ is $1 / M$ times that of $f(\boldsymbol{D})$, since any single observation appears in at most one of the partitions. Finally, we use the Laplace mechanism to release a noisy version of $M^{-1} \sum_{l=1}^{M} f\left(\boldsymbol{D}_{l}\right)$.

\section{The Differentially Private Test Statistic}

We begin by laying out relevant notation and formally specifying our objectives. Let $\boldsymbol{D}$ be a confidential dataset comprising $n$ individuals. For each individual $i=1, \ldots, n$, let $y_{i} \in \mathbb{R}$ be its univariate response variable and $x_{i}=\left(1, x_{i, 1}, \ldots, x_{i, p}\right)^{\top} \in \mathbb{R}^{p+1}$ be its $(p+1) \times 1$ vector of predictors. Hence, $\boldsymbol{D}=\left\{\left(x_{i}, y_{i}\right)\right\}_{i=1}^{n}$. An analyst seeks to estimate the parameters in the regression, $y_{i}=\boldsymbol{\beta}^{\top} x_{i}+e_{i}$, where $\boldsymbol{\beta}=\left(\beta_{0}, \ldots, \beta_{p}\right)^{\top} \in \mathbb{R}^{p+1}$ and $e_{i}$ are i.i.d. random errors with $E\left(e_{i}\right)=0$ and $\operatorname{Var}\left(e_{i}\right)=\sigma^{2}$. In linear regression, we typically assume that $e_{i} \sim N\left(0, \sigma^{2}\right)$ for all $i$, although our algorithm can be used with other error distributions. We 
assume that, if the analyst had direct access to $D$, he or she would make inferences about each $\beta_{j}$ based on the maximum likelihood estimator (MLE), $\hat{\beta}_{j}$, and its corresponding sampling distribution. However, the analyst does not get direct access to $D$; instead, the analyst can learn only the privately-computed estimates $\tilde{\beta}_{j}$, which could arise from perturbed versions of $\hat{\beta}_{j}$ or from synthetic data. Our key question is whether or not inferences about $\beta_{j}$ are similar when using $\hat{\beta}_{j}$ or $\tilde{\beta}_{j}$.

To address this question, we develop differentially private significance tests. Let $T(\boldsymbol{D})$ be the standardized estimator of $\beta_{j}$ obtained from $D$, that is,

$$
T(\boldsymbol{D})=\hat{\beta}_{j} / \sqrt{\hat{\Sigma}_{j, j}}
$$

where $\left(\hat{\Sigma}_{j, j}\right)$ is the $(j, j)$ th element of the matrix, $\hat{\Sigma}=\hat{\sigma}^{2}\left(\mathbf{X}_{\boldsymbol{D}}^{\top} \mathbf{X}_{\boldsymbol{D}}\right)^{-1}$. Here, $\hat{\sigma}^{2}=\left(\mathbf{y}_{\boldsymbol{D}}-\right.$ $\left.\hat{\boldsymbol{\beta}}^{\top} \mathbf{X}_{\boldsymbol{D}}\right)^{\top}\left(\mathbf{y}_{\boldsymbol{D}}-\hat{\boldsymbol{\beta}}^{\top} \mathbf{X}_{\boldsymbol{D}}\right) /(n-p-1)$, where $\mathbf{y}_{\boldsymbol{D}}=\left(y_{1}, \ldots, y_{n}\right)^{\top}$, and $\mathbf{X}_{\boldsymbol{D}}=\left[x_{1}^{\top}, \ldots, x_{n}^{\top}\right]^{\top}$ is the design matrix associated with the regression when estimated with $\boldsymbol{D}$. Under certain conditions on $\mathbf{X}_{\boldsymbol{D}}, \hat{\beta}_{j}$ is asymptotically normally distributed for a large range of error distributions $\operatorname{Van}$ der Vaart, 2000, page 21; Bhattacharya et al., 2016, section 6.8). The key condition on the design is that the maximum among the diagonal elements of the matrix $\mathbf{X}_{D}\left(\mathbf{X}_{D}^{\top} \mathbf{X}_{D}\right)^{-1} \mathbf{X}_{D}^{\top}$ goes to zero as $n \rightarrow \infty$. Hence, for a large enough $n$, the distribution of $T(\boldsymbol{D})$ can be suitably approximated by a standard Gaussian distribution. In the remainder of the article, we refer to $T(\boldsymbol{D})$ simply as the $t$-statistic.

$T(\boldsymbol{D})$ provides all the information needed for inferences about the sign and significance of $\beta_{j}$. Hence, we can address our key question and account for privacy by developing algorithms for releasing differentially private versions of $T(\boldsymbol{D})$, along with deriving reference distributions for the private $t$-statistics. Taken together, these algorithms enable the analyst to assess the significance level for $\beta_{j}$ directly from the private output.

Unfortunately, we cannot simply apply the Laplace mechanism in (1) to create the differentially private test statistic, as the global sensitivity of $T(\boldsymbol{D})$ is unbounded. A possible remedy is 
to work with some bounded statistic instead of $T(\boldsymbol{D})$. For statistical significance, two obvious candidates include (i) the p-value associated with $T(\boldsymbol{D})$ and (ii) a truncated version of $T(\boldsymbol{D})$. We next describe some of the pros and cons of each approach.

Let $p^{T}$ be the p-value associated with $T(\boldsymbol{D})$ for a two-tailed significance test of the null hypothesis $\beta_{j}=0$. Any $p^{T}$ has global sensitivity equal to one. Let $p^{T, \epsilon}$ be the $\epsilon$-differentially private $\mathrm{p}$-value obtained after adding Laplace noise to $p^{T}$ based on the global sensitivity of one. With high probability, adding this noise to small values of $p^{T}$ could inflate them so much as to change our opinion of the significance of $\beta_{j}$. This is less problematic when adding noise to large values of $p^{T}$. In other words, for a given $\epsilon$, the probability that an analyst reaches the same decisions about statistical significance when using $p^{T, \epsilon}$ or $p^{T}$ is higher when $p^{T}$ falls in an acceptance region for $H_{0}$ than when $p^{T}$ falls in a rejection region.

Regarding the second approach, let the truncated $t$-statistic be given by

$$
T^{t}(\boldsymbol{D})= \begin{cases}-a & \text { if } T(\boldsymbol{D})<-a \\ T(\boldsymbol{D}) & \text { if }-a \leq T(\boldsymbol{D}) \leq a \\ a & \text { if } T(\boldsymbol{D})>a\end{cases}
$$

where $a>0$ is a user-defined parameter. Here, $a$ has to be large enough to ensure that $T(\boldsymbol{D})$ and $T^{t}(\boldsymbol{D})$ lead to the same conclusion regarding the null hypothesis with high probability. Because of the truncation, the global sensitivity of $T^{t}(\boldsymbol{D})$ equals $2 a$. Let $T^{t, \epsilon}(\boldsymbol{D})=T^{t}(\boldsymbol{D})+\eta$ be a noisy version of $T^{t}(\boldsymbol{D})$, where $\eta \sim \operatorname{Lap}(0,2 a / \epsilon)$ and $\operatorname{Lap}(l, s)$ denotes the Laplace distribution with location $l$ and scale $s$. The problematic situations for $T^{t, \epsilon}(\boldsymbol{D})$ are the reverse of those for $p^{T}$. With undesirably high probability, adding noise to values of $T^{t}(\boldsymbol{D})$ near zero could make an insignificant effect appear significant, whereas the noise is not likely to change our opinion about significance when $T(\boldsymbol{D})$ is large. Put another way, for a given $\epsilon$, the probability that an analyst reaches the same decisions about statistical significance when using $T^{t, \epsilon}(\boldsymbol{D})$ or $T^{t}(\boldsymbol{D})$ is higher when $T^{t}(\boldsymbol{D})$ falls in a rejection region for $H_{0}$ than when $T^{t}(\boldsymbol{D})$ falls in an acceptance 
region.

The arguments above suggest that neither approach always outperforms the other. We opt for the second approach because $T^{t}(\boldsymbol{D})$ is more analytically tractable than $p^{T}$. As a result, we find it easier to develop a properly calibrated significance test, and understand its theoretical properties, for $T^{t}(\boldsymbol{D})$ than for $p^{T}$. Using $T^{t}(\boldsymbol{D})$ also allows the release of a noisy estimate of the sign of $\beta_{j}$ without additional expenditure of $\epsilon$, which improves the overall utility of the data release without sacrificing privacy.

Since the length of the range of $T^{t}(\boldsymbol{D})$ coincides with its global sensitivity, we need to use a large $\epsilon$ to ensure that $T^{t, \epsilon}(\boldsymbol{D})$ is practically useful, perhaps larger than what we would like from the perspective of protecting privacy. Put another way, for a small $\epsilon$ the noise introduced by the Laplace mechanism may be so large compared to $T^{t}(\boldsymbol{D})$ that the statistic has little ability to detect any deviations from the null hypothesis. Hence, we need to adapt the truncated $t$-statistic to reduce the global sensitivity.

We consider a way to do so based on the subsample and aggregate method described in Section 2. We first randomly partition $\boldsymbol{D}$ into $M$ disjoint subsets, $\mathcal{P}=\left\{\boldsymbol{D}_{1}, \ldots, \boldsymbol{D}_{M}\right\}$, of equal size (or as close to equal as possible when $n / M$ is not an integer). In each $\boldsymbol{D}_{l}$, we estimate the regression model of interest using only $\boldsymbol{D}_{l}$. For the regression coefficient of interest, we then compute the set of $M$ t-statistics, $\left\{T\left(\boldsymbol{D}_{1}\right), \ldots, T\left(\boldsymbol{D}_{M}\right)\right\}$, and truncate each $T\left(\boldsymbol{D}_{l}\right)$ at $[-a, a]$, akin to 22. Let $\left\{T^{t}\left(\boldsymbol{D}_{1}\right), \ldots, T^{t}\left(\boldsymbol{D}_{M}\right)\right\}$ be the set of $M$ truncated $t$-statistics. We then compute

$$
\bar{T}^{t}(\mathcal{P})=\sum_{l=1}^{M} T^{t}\left(\boldsymbol{D}_{l}\right) / M
$$

The sensitivity of $\bar{T}^{t}(\mathcal{P})$ is $1 / M$ times the sensitivity of $T^{t}(\boldsymbol{D})$, which apparently achieves our goal. However, we do not create the differentially private measure by adding Laplace noise to $\bar{T}^{t}(\mathcal{P})$, for reasons we now describe.

Because of the random partitioning, it is reasonable to consider each $\boldsymbol{D}_{l}$ as a random sample from a population (with infinite sample size) and, thus, each $T^{t}\left(\boldsymbol{D}_{l}\right)$ as a random draw from 

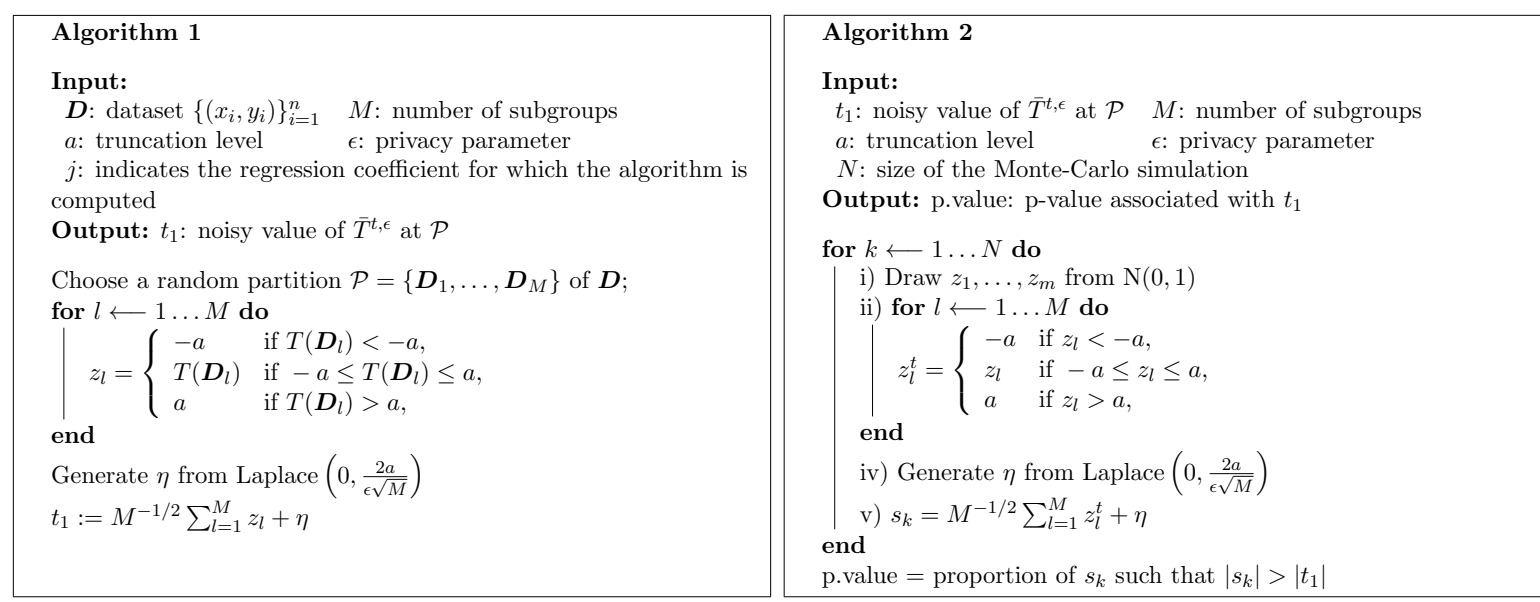

Figure 1: Differentially private algorithms for $\bar{T}^{t, \epsilon}$. Algorithm 1 shows the steps to release values of $\bar{T}^{t, \epsilon}$, for a given $(M, a, \epsilon)$. Algorithm 2 shows how to compute the p-value associated with the released $t$-statistics of Algorithm 1.

its sampling distribution. We would like the sampling distributions of $T^{t}(\boldsymbol{D})$ and $\bar{T}^{t}(\mathcal{P})$ to be approximately the same, so that the significance test based on $\bar{T}^{t}(\mathcal{P})$ would have approximately the same power function as that based on $T^{t}(\boldsymbol{D})$. When this is the case, analysts should have high probability of reaching similar conclusions when using the adapted $t$-statistic or $T^{t}(\boldsymbol{D})$. However, the variance of $\bar{T}^{t}(\mathcal{P})$ is roughly $M$ times smaller than the variance of $T^{t}(\boldsymbol{D})$. Thus, instead of using $\bar{T}^{t}(\mathcal{P})$ directly, we equate the variances by multiplying $\bar{T}^{t}(\mathcal{P})$ by $\sqrt{M}$; that is, we use $\bar{T}^{t, R}(\mathcal{P})=\sqrt{M} \bar{T}^{t}(\mathcal{P})$. This changes the global sensitivity, as it increases from $2 a / M$ to $2 a / \sqrt{M}$. Hence, the differentially private version of the $t$-statistic is $\bar{T}^{t, \epsilon}(\mathcal{P})=\bar{T}^{t, R}(\mathcal{P})+\eta$, where $\eta \sim \operatorname{Lap}(0,2 a / \sqrt{M} \epsilon)$.

Figure 1 displays the steps that agencies can use to release $\bar{T}^{t, \epsilon}(\mathcal{P})$ (Algorithm 1). The figure also describes simple Monte Carlo algorithms that can be used to approximate the sampling distribution of $\bar{T}^{t, \epsilon}(\mathcal{P})$ (Algorithm 2). This reference distribution can be used to obtain approximate p-values corresponding to the test statistic. The Monte Carlo simulations are needed to properly account for all sources of randomness, including the noise from the Laplace Mechanism. Taken together, Algorithm 1 and 2 provide a means to perform differentially private significance tests. Inferences about the sign of $\beta_{j}$ can be obtained from the sign of $\bar{T}^{t, \epsilon}(\mathcal{P})$. 
Finally, we conclude this section with a formal theorem and proof that Algorithm 1 is differentially private.

Theorem 1. Algorithm 1 satisfies $\epsilon$-differential privacy.

Proof. $\bar{T}^{t, R}$ has global sensitivity equal to $2 a / \sqrt{M}$. Hence, defining $\bar{T}^{t, \epsilon}(\mathcal{P})=\bar{T}^{t, R}(\mathcal{P})+$ $\operatorname{Lap}(2 a / \sqrt{M} \epsilon)$ implies that, by Definition 1, Algorithm 1 satisfies $\epsilon$-differential privacy.

\section{Theoretical Properties}

In this section, we discuss some of the theoretical properties of $\bar{T}^{t, \epsilon}(\mathcal{P})$. First, we derive conditions that characterize the distance between $\bar{T}^{t, \epsilon}(\mathcal{P})$ and $T(\boldsymbol{D})$. We then study the asymptotic probability of type II errors for the statistical test defined from $\bar{T}^{t, \epsilon}(\mathcal{P})$.

\subsection{Distance between $\bar{T}^{t, \epsilon}(\mathcal{P})$ and $T(\boldsymbol{D})$}

To characterize the distance between $\bar{T}^{t, \epsilon}(\mathcal{P})$ and $T(\boldsymbol{D})$, we focus on the probability $P\left\{\left|\bar{T}^{t, \epsilon}(\mathcal{P})-T(\boldsymbol{D})\right|>c \mid \mathbf{X}_{\boldsymbol{D}}, \mathcal{R}\right\}$ for all $c>0$, where $\mathcal{R}$ denotes the random mechanism used to partition $\boldsymbol{D}$ and to create $\mathcal{P}$. We note that $\boldsymbol{D}$ and $\mathcal{P}$ are functions of $\left(\mathbf{y}_{\boldsymbol{D}}, \mathbf{X}_{\boldsymbol{D}}\right)$ and $\left(\mathbf{y}_{\boldsymbol{D}}, \mathbf{X}_{\boldsymbol{D}}, \mathcal{R}\right)$, respectively. Since we are conditioning on $\mathbf{X}_{\boldsymbol{D}}$ and $\mathcal{R}$, the randomness in $T(\boldsymbol{D})$ and $\bar{T}^{t, \epsilon}(\mathcal{P})$ comes from treating $\mathbf{y}_{\boldsymbol{D}}$ as a random variable. We use the triangle inequality to bound this probability and, in this way, focus on characterizing each of the terms defining the right hand-side of

$$
\begin{aligned}
P\left\{\left|\bar{T}^{t, \epsilon}(\mathcal{P})-T(\boldsymbol{D})\right|>c \mid \mathbf{X}_{\boldsymbol{D}}, \mathcal{R}\right\} \leq & P\left\{\left|\bar{T}^{t, \epsilon}(\mathcal{P})-\bar{T}^{t, R}(\mathcal{P})\right|>c \mid \mathbf{X}_{\boldsymbol{D}}, \mathcal{R}\right\} \\
& +P\left\{|\sqrt{M} \bar{T}(\mathcal{P})-T(\boldsymbol{D})|>c \mid \mathbf{X}_{\boldsymbol{D}}, \mathcal{R}\right\} \\
& +P\left\{\left|\bar{T}^{t, R}(\mathcal{P})-\sqrt{M} \bar{T}(\mathcal{P})\right|>c \mid \mathbf{X}_{\boldsymbol{D}}, \mathcal{R}\right\}
\end{aligned}
$$


where $\bar{T}(\mathcal{P})=M^{-1} \sum_{l=1}^{M} T\left(\boldsymbol{D}_{l}\right)$. The first term is relatively straightforward to understand theoretically and corresponds to computing a probability under the Laplace distribution. Thus,

$$
P\left\{\left|\bar{T}^{t, \epsilon}(\mathcal{P})-\bar{T}^{t, R}(\mathcal{P})\right|>c \mid \mathbf{X}_{\boldsymbol{D}}, \mathcal{R}\right\}=\exp \left(-\frac{c \epsilon \sqrt{M}}{2 a}\right)
$$

As expected, the bound in (4) indicates that $\bar{T}^{t, \epsilon}(\mathcal{P})$ gets closer to $\bar{T}^{t, R}(\mathcal{P})$ as the scale of the underlying Laplace distribution, $2 a / \epsilon \sqrt{M}$, goes to zero, that is, as $M$ and $\epsilon$ increase and $a$ decreases.

Turning to the second term in (3), we now provide conditions that ensure $\sqrt{M} \bar{T}(\mathcal{P})$ is a reasonable approximation of $T(\boldsymbol{D})$. To begin, we treat each $\boldsymbol{D}_{l}$ as an independent sample from an infinite population. We make inferences conditional on each $\mathbf{X}_{\boldsymbol{D}_{l}}$. Throughout, we rely on the following assumption.

Assumption A1. The distribution of $\hat{\beta}_{j l}$, i.e., the MLE of $\beta_{j}$ estimated with $\boldsymbol{D}_{l}$ treating $\mathbf{X}_{\boldsymbol{D}_{l}}$ as fixed, can be suitably approximated by a Gaussian distribution with mean $\beta_{j}$ and variance $\Sigma_{j, j}\left(\boldsymbol{D}_{l}\right)$, where $\Sigma_{j, j}\left(\boldsymbol{D}_{l}\right)$ denotes the $j$ th diagonal element of $\Sigma\left(\boldsymbol{D}_{l}\right)=\sigma^{2}\left(\mathbf{X}_{\boldsymbol{D}_{l}}^{T} \mathbf{X}_{\boldsymbol{D}_{l}}\right)^{-1}$ and $\mathbf{X}_{D_{l}}$ is the design matrix associated with $\boldsymbol{D}_{l}$.

A1 is widely assumed in practice in regression modeling (see Van der Vaart, 2000, page 21; Bhattacharya et al., 2016, section 6.8). Under A1, treating each $\boldsymbol{D}_{l}$ as independent samples and conditioning on $\mathbf{X}_{\boldsymbol{D}_{l}}$ implies that $\sqrt{M} \bar{T}(\mathcal{P})$ and $T(\boldsymbol{D})$ are both Gaussian-distributed with variance equal to one. However, it is not necessarily the case that the means of each $T\left(\boldsymbol{D}_{l}\right)$ are equal just because the conditional means of each $\hat{\beta}_{j l}$ are equal, nor that these means equal the mean of $T(\boldsymbol{D})$. In particular, when $\beta_{j} \neq 0, T\left(\boldsymbol{D}_{l}\right)$ is based on a smaller sample size and a different design matrix than $T(\boldsymbol{D})$, which results in different (typically smaller) expected values. Thus, we need to derive conditions on the means of each $\sqrt{M} T\left(\boldsymbol{D}_{l}\right)$, where $l=1, \ldots, M$, that guarantee the mean of $\sqrt{M} \bar{T}(\mathcal{P})$ is close to the mean of $T(\boldsymbol{D})$. 
Since each $\boldsymbol{D}_{l}$ in actuality is a random sample from $\boldsymbol{D}$, as long as the sample size in each partition is large it is reasonable to assume that $\left(\mathbf{X}_{\boldsymbol{D}_{l}}^{\top} \mathbf{X}_{\boldsymbol{D}_{l}}\right) \approx\left(\mathbf{X}_{\boldsymbol{D}_{k}}^{\top} \mathbf{X}_{\boldsymbol{D}_{k}}\right)$ for all pairs of datasets $(l, k)$. Moreover, it also is reasonable to make the following assumption.

Assumption A2. $M\left(\mathbf{X}_{D_{l}}^{\top} \mathbf{X}_{D_{l}}\right) \approx\left(\mathbf{X}_{D}^{\top} \mathbf{X}_{D}\right)$ for all $l=1, \ldots, M$.

With $\mathbf{A 2}$, we have $M^{-1} \Sigma\left(\boldsymbol{D}_{l}\right) \approx \Sigma(\boldsymbol{D})$. Using this approximation, we take expectations of $\sqrt{M} \bar{T}(\mathcal{P})$ conditional on the realized $\left(\mathbf{X}_{\boldsymbol{D}}, \mathcal{R}\right)$. We have

$$
\begin{aligned}
E\left\{\sqrt{M} \bar{T}(\mathcal{P}) \mid \mathbf{X}_{\boldsymbol{D}}, \mathcal{R}\right\} & =M^{-1} \sum_{l=1}^{M} E\left\{\sqrt{M} T\left(\boldsymbol{D}_{l}\right) \mid \mathbf{X}_{\boldsymbol{D}}, \mathcal{R}\right\} \\
& =M^{-1} \sum_{l=1}^{M} \sqrt{M} \beta_{j} / \sqrt{\Sigma_{j, j}\left(\boldsymbol{D}_{l}\right)} \\
& \approx M^{-1} \sum_{l=1}^{M} \beta_{j} / \sqrt{\Sigma_{j, j}(\boldsymbol{D})}=E\left\{T(\boldsymbol{D}) \mid \mathbf{X}_{\boldsymbol{D}}\right\}
\end{aligned}
$$

Additionally, using the approximation in $\mathbf{A 2}$, for any $\boldsymbol{D}_{l}$ the

$$
\operatorname{Cov}\left\{\left(\mathbf{X}_{\boldsymbol{D}}^{\top} \mathbf{X}_{\boldsymbol{D}}\right)^{-1} \mathbf{X}_{\boldsymbol{D}}^{\top} \mathbf{y}_{\boldsymbol{D}},\left(\mathbf{X}_{\boldsymbol{D}_{l}}^{\top} \mathbf{X}_{\boldsymbol{D}_{l}}\right)^{-1} \mathbf{X}_{\boldsymbol{D}_{l}}^{\top} \mathbf{y}_{\boldsymbol{D}_{l}} \mid \mathbf{X}_{\boldsymbol{D}}, \mathcal{R}\right\}=\sigma^{2}\left(\mathbf{X}_{\boldsymbol{D}}^{\top} \mathbf{X}_{\boldsymbol{D}}\right)^{-1}=\Sigma(\boldsymbol{D}) .
$$

Therefore, we have

$$
\begin{aligned}
\operatorname{Cov}\left\{T(\boldsymbol{D}), \sqrt{M} \bar{T}(\mathcal{P}) \mid \mathbf{X}_{\boldsymbol{D}}, \mathcal{R}\right\} & =\frac{\sqrt{M}}{M} \sum_{l=1}^{M} \operatorname{Cov}\left\{T(\boldsymbol{D}), T\left(\boldsymbol{D}_{l}\right) \mid \mathbf{X}_{\boldsymbol{D}}, \mathcal{R}\right\} \\
& =\frac{1}{M} \sum_{l=1}^{M} \frac{\sqrt{\Sigma_{j, j}(\boldsymbol{D})}}{\sqrt{M^{-1} \Sigma_{j, j}\left(\boldsymbol{D}_{l}\right)}}>0 .
\end{aligned}
$$

Thus, the smaller the distance is between $\mathbf{X}_{\boldsymbol{D}}^{\top} \mathbf{X}_{\boldsymbol{D}}$ and $M\left(\mathbf{X}_{\boldsymbol{D}_{l}}^{\top} \mathbf{X}_{\boldsymbol{D}_{l}}\right)$, the higher is the correlation between $\sqrt{M} \bar{T}(\mathcal{P})$ and $T(\boldsymbol{D})$. 
A direct application of the Markov inequality implies that

$$
\begin{aligned}
c^{2} P\left\{|\sqrt{M} \bar{T}(\mathcal{P})-T(\boldsymbol{D})|>c \mid \mathbf{X}_{\boldsymbol{D}}, \mathcal{R}\right\} \leq \\
\operatorname{Var}\left\{\sqrt{M} \bar{T}(\mathcal{P}) \mid \mathbf{X}_{\boldsymbol{D}}, \mathcal{R}\right\}+\operatorname{Var}\left\{T(\boldsymbol{D}) \mid \mathbf{X}_{\boldsymbol{D}}, \mathcal{R}\right\}-2 \operatorname{Cov}\left\{T(\boldsymbol{D}), \sqrt{M} \bar{T}(\mathcal{P}) \mid \mathbf{X}_{\boldsymbol{D}}, \mathcal{R}\right\} \\
+\left[E\left\{T(\boldsymbol{D}) \mid \mathbf{X}_{\boldsymbol{D}}, \mathcal{R}\right\}-E\left\{\sqrt{M} \bar{T}(\mathcal{P}) \mid \mathbf{X}_{\boldsymbol{D}}, \mathcal{R}\right\}\right]^{2} .
\end{aligned}
$$

Thus, under A2, by $\mathbf{A} 1$ and (5) we have

$$
\operatorname{Var}\left\{\sqrt{M} \bar{T}(\mathcal{P}) \mid \mathbf{X}_{\boldsymbol{D}}, \mathcal{R}\right\} \approx \operatorname{Var}\left\{T(\boldsymbol{D}) \mid \mathbf{X}_{\boldsymbol{D}}, \mathcal{R}\right\} \approx 1
$$

and

$$
E\left\{T(\boldsymbol{D}) \mid \mathbf{X}_{\boldsymbol{D}}, \mathcal{R}\right\} \approx E\left\{\sqrt{M} \bar{T}(\mathcal{P}) \mid \mathbf{X}_{\boldsymbol{D}}, \mathcal{R}\right\}
$$

By (6), we have

$$
\operatorname{Cov}\left\{T(\boldsymbol{D}), \sqrt{M} \bar{T}(\mathcal{P}) \mid \mathbf{X}_{\boldsymbol{D}}, \mathcal{R}\right\} \approx 1
$$

Therefore, the probability in (7) is near zero, implying that the distance between $\sqrt{M} \bar{T}(\mathcal{P})$ and $T(\boldsymbol{D})$ has high probability of being small.

Finally, we provide an upper bound for the last term in (3). This bound allows us to understand how choices of $M$ and $a$ affect the distance between $\sqrt{M} \bar{T}(\mathcal{P})$ and $\bar{T}^{t, R}(\mathcal{P})$. Specifically, it follows that

$$
\begin{aligned}
P\left\{\left|\sqrt{M} \bar{T}(\mathcal{P})-\bar{T}^{t, R}(\mathcal{P})\right|>c \mid \mathbf{X}_{\boldsymbol{D}}, \mathcal{R}\right\} & \leq P\left\{\left|\sqrt{M} \bar{T}(\mathcal{P})-\bar{T}^{t, R}(\mathcal{P})\right|>0 \mid \mathbf{X}_{\boldsymbol{D}}, \mathcal{R}\right\} \\
& =1-P\left\{\sqrt{M} \bar{T}(\mathcal{P})=\bar{T}^{t, R}(\mathcal{P}) \mid \mathbf{X}_{\boldsymbol{D}}, \mathcal{R}\right\} \\
& \leq 1-\prod_{l=1}^{M} P\left\{T\left(\boldsymbol{D}_{l}\right)=T^{t}\left(\boldsymbol{D}_{l}\right) \mid \mathbf{X}_{\boldsymbol{D}}, \mathcal{R}\right\} \\
& =1-(\Phi(a-\mu)-\Phi(-a-\mu))^{M}
\end{aligned}
$$

where $\mu=E\left\{T\left(\boldsymbol{D}_{l}\right) \mid \mathbf{X}_{\boldsymbol{D}_{l}}, \mathcal{R}\right\}$ and $\Phi$ denotes the cumulative distribution function of the stan- 
dard Gaussian distribution. The probability in $(8)$ reveals that $\bar{T}^{t, R}(\mathcal{P})$ gets closer to $\sqrt{M} \bar{T}(\mathcal{P})$ as $a$ increases and $M$ decreases. Together, (4)-(8) provide a full characterization of (3).

\subsection{Asymptotic power properties of $\bar{T}^{t, \epsilon}(\mathcal{P})$}

We next study the type II error rates for the significance test defined from $\bar{T}^{t, \epsilon}(\mathcal{P})$. For given values of $(M, a, \epsilon)$, and under $H_{0}: \beta_{j}=0$ (i.e., $\mu=0$ ), let $r$ be a positive constant such that

$$
P_{H_{0}}\left\{\left|\bar{T}^{t, \epsilon}(\mathcal{P})\right|<r \mid \mathbf{X}_{\boldsymbol{D}}, \mathcal{R}\right\}=1-\alpha
$$

where $P_{H_{0}}$ denotes the probability computed under $H_{0}$. Here, $r$ corresponds to the critical value that ensures a significance level of $\alpha$ for the test $\mathbb{T}_{t, \epsilon}(\mathcal{P})=\mathbb{I}_{(-r, r)}\left(\bar{T}^{t, \epsilon}(\mathcal{P})\right)$, where $\mathbb{I}_{B}(b)=1$ if $b \in B$ and $\mathbb{I}_{B}(b)=0$ otherwise. Thus, the type II error probability associated with this test is given by $E_{H_{1}}\left\{\mathbb{T}_{t, \epsilon}(\mathcal{P}) \mid \mathbf{X}_{\boldsymbol{D}}, \mathcal{R}\right\}$, where $E_{H_{1}}$ denotes expectation under $H_{1}: \beta_{j} \neq 0$.

The strategy used to define $\bar{T}^{t, \epsilon}(\mathcal{P})$ makes it difficult to derive analytical expressions for $r$ and $E_{H_{1}}\left\{\mathbb{T}_{t, \epsilon}(\mathcal{P}) \mid \mathbf{X}_{\boldsymbol{D}}, \mathcal{R}\right\}$ in terms of $(\alpha, M, a, \epsilon)$. However, since $\bar{T}^{t, \epsilon}(\mathcal{P})$ is a function of random variables that are easy to generate numerically, it is trivial to use Monte Carlo simulation to provide accurate approximations of $r$ and $E_{H_{1}}\left\{\mathbb{T}_{t, \epsilon}(\mathcal{P}) \mid \mathbf{X}_{\boldsymbol{D}}, \mathcal{R}\right\}$. This allows us assess type II error rates for the test both asymptotically, which we study in this section, and in finite samples, which we study in Section 5 .

As $n$ goes to infinity, $\bar{T}^{t, R}(\mathcal{P})$ converges in probability to $\sqrt{M} a$. Hence, the asymptotic probability of type II error is approximately equal to the probability of the acceptance region $(-r, r)$ under a Laplace distribution with location and scale equal to $\sqrt{M} a$ and $2 a / \sqrt{M} \epsilon$, respectively. This characterization immediately reveals that the probability of type II error for this test never equals zero.

Figure 2 displays a Monte Carlo approximation of the asymptotic probability of type II error associated with the test $\mathbb{T}_{t, \epsilon}(\mathcal{P})$ for different values of $(\alpha, a, M, \epsilon)$. For most of the combina- 
tions of $(\alpha, M, a, \epsilon)$ studied here, it is possible to obtain an asymptotic probability of type II error that is close to zero, with $(\alpha=0.01, \epsilon=0.1)$ being the lone exception. For any given $(\alpha, M, \epsilon)$, the asymptotic probability of type II error is almost constant as a function of $a$, that is, $\lim _{n \rightarrow \infty} E_{H_{1}}\left\{\mathbb{T}_{t, \epsilon}(\mathcal{P}) \mid \mathbf{X}_{\boldsymbol{D}}, \mathcal{R}\right\}$ decreases at a slow rate as $a$ increases. The type II error probabilities also decrease as any one of $M, \alpha$, or $\epsilon$ increases, holding the others constant. We note that analysts can use this Monte Carlo approach to approximate the asymptotic probability of type II error for any combination of $(\alpha, M, a, \epsilon)$. Thus, for example, for a fixed $(\alpha, \epsilon)$, analysts can determine values of $(M, a)$ that lead to a specified asymptotic probability of type II error. For example, for $\alpha=0.05, \epsilon=1$, and $\lim _{n \rightarrow \infty} E_{H_{1}}\left\{\mathbb{T}_{t, \epsilon}(\mathcal{P}) \mid \mathbf{X}_{\boldsymbol{D}}, \mathcal{R}\right\}<0.001$, we find that $M$ needs to be greater than 25 provided that $a$ is greater than one.

Although the Monte Carlo approach provides accurate and straightforward approximations, it is also instructive to characterize the asymptotic behavior of $E_{H_{1}}\left\{\mathbb{T}_{t, \epsilon}(\mathcal{P}) \mid \mathbf{X}_{\boldsymbol{D}}, \mathcal{R}\right\}$ mathematically under arbitrary choices of $(\alpha, a, M, \epsilon)$. Theorem 2 provides an upper bound for $\lim _{n \rightarrow \infty} E_{H_{1}}\left\{\mathbb{T}_{t, \epsilon}(\mathcal{P}) \mid \mathbf{X}_{\boldsymbol{D}}, \mathcal{R}\right\}$. Analogous to Figure 2, in the supplementary material we present a graphical representation of the upper bound. We observe that $(9)$ is a sharp bound when $a>2$. For $1 \leq a \leq 2$ and some values of $M$, the bound in (9) is a moderately less precise, but still valid, bound for $\lim _{n \rightarrow \infty} E_{H_{1}}\left\{\mathbb{T}_{t, \epsilon}(\mathcal{P}) \mid \mathbf{X}_{\boldsymbol{D}}, \mathcal{R}\right\}$. The proof of Theorem 2 is in the supplementary material.

Theorem 2. Under $H_{1}: \beta_{j} \neq 0$ and assumption A1,

$$
\begin{aligned}
\lim _{n \rightarrow \infty} E\left\{\mathbb{T}_{t, \epsilon} \mid \mathbf{X}_{\boldsymbol{D}}, \mathcal{P}\right\} & <\mathbb{I}_{\left\{r^{*}<\sqrt{M} a\right\}} \frac{1}{2}\left(\alpha^{-1-\epsilon \sqrt{M} / 2 a}-\alpha^{1+\epsilon \sqrt{M} / 2 a}\right) \exp \left(-\frac{\epsilon M}{2}\right) \\
+ & \mathbb{I}_{\left\{r^{*}>\sqrt{M} a\right\}}\left(1-\frac{1}{2} \alpha^{1+\epsilon \sqrt{M} / 2 a}\left[\exp \left(\frac{\epsilon M}{2}\right)-\exp \left(-\frac{\epsilon M}{2}\right)\right]\right)
\end{aligned}
$$

where $r^{*}=-\log (\alpha)(2 a / \epsilon \sqrt{M}+1)$. 

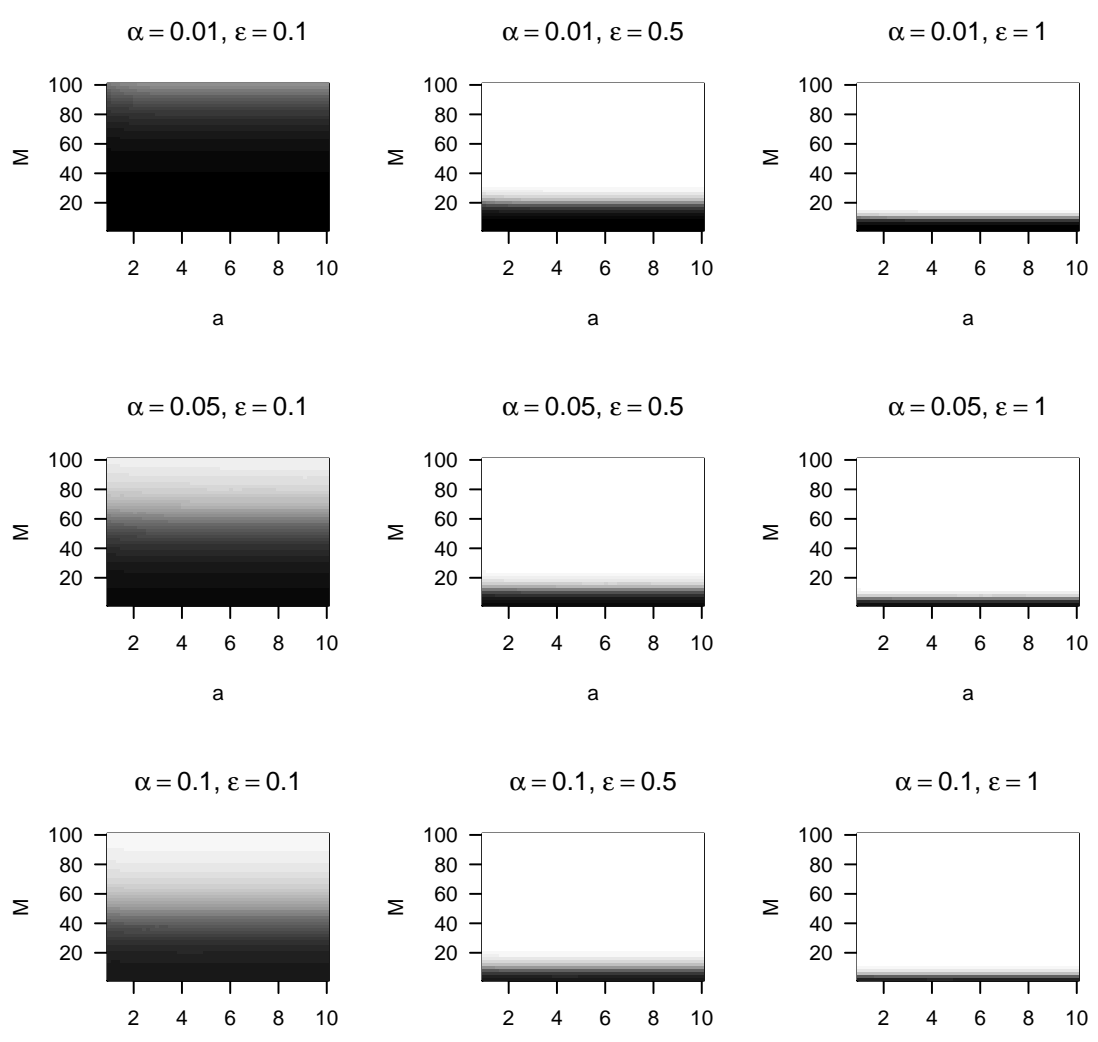

a

Figure 2: Asymptotic probability of type II error associated with the test $\mathbb{T}_{t, \epsilon}(\mathcal{P})$ for different values of $(\alpha, a, M, \epsilon)$. Each asymptotic probability is computed using Monte Carlo approximation based on 10,000 realizations of $\mathbb{T}_{t, \epsilon}(\mathcal{P})$. Within any panel and combination $(M, a)$, black represents $\lim _{n \rightarrow \infty} E_{H_{1}}\left\{\mathbb{T}_{t, \epsilon}(\mathcal{P}) \mid \mathbf{X}_{\boldsymbol{D}}, \mathcal{R}\right\}=1$ and white represents $\lim _{n \rightarrow \infty} E_{H_{1}}\left\{\mathbb{T}_{t, \epsilon}(\mathcal{P}) \mid \mathbf{X}_{\boldsymbol{D}}, \mathcal{R}\right\}=0$, with lighter grays as the loss function approaches 0 .

\section{Empirical Illustrations}

The results in Section 4.1 suggest we should make $a$ as large as possible and $M$ as small as possible to ensure the proximity between $\bar{T}^{t, R}(\mathcal{P})$ and $T(\boldsymbol{D})$. However, the accuracy of the approximation of $T(\boldsymbol{D})$ is only part of the story. We need to add Laplace noise to protect privacy. To maximize the usefulness of the privately-computed $t$-statistic $\bar{T}^{t, \epsilon}(\mathcal{P})$, we seek to add as little noise as possible while still satisfying differential privacy. This pushes us to make $a$ smaller rather than larger, and to make $M$ larger rather than smaller. How do we trade off accuracy in $\bar{T}^{t, R}(\mathcal{P})$ for reductions in variance of the Laplace noise? We have a partial answer 
this question from the asymptotic results in Section 4.2 , but, in practice, we have to analyze finite samples. Which choices of $(M, a)$ tend to offer higher accuracy for a given risk level $\epsilon$ with finite samples?

In this section, we address this question using simulation studies. In all simulations, we assume that $\hat{\beta}_{j} \sim \mathrm{N}\left(\beta_{j}, \Sigma_{j, j}(\boldsymbol{D})\right)$ and $\hat{\beta}_{j, l} \sim \mathrm{N}\left(\beta_{j}, \Sigma_{j, j}\left(\boldsymbol{D}_{l}\right)\right)$ for any $j$ and $l$, i.e., we assume that $\mathbf{A 1}$ holds. We consider two scenarios, one where $M \mathbf{X}_{\boldsymbol{D}_{l}}^{\top} \mathbf{X}_{\boldsymbol{D}_{l}} \approx\left(\mathbf{X}_{\boldsymbol{D}}^{\top} \mathbf{X}_{\boldsymbol{D}}\right)$, where $l=1, \ldots, M$, i.e., where $\mathbf{A} \mathbf{2}$ holds, and the other where this is not necessarily the case. The scenarios are generated as follows.

In Scenario I, we work directly with the theoretical distributions of the $t$-statistics, without simulating and partitioning values of $\boldsymbol{D}$. For an arbitrary regression coefficient $\beta$, let $\mu_{T}$ be the number of standard deviations that its value is from zero. We consider multiple values of $\mu_{T}$ in the simulation. For any $\mu_{T}$, we generate $T$ and $\bar{T}^{t, R}$ from their sampling distributions as follows,

$$
\begin{aligned}
T & \sim \mathrm{N}\left(\mu_{T}, 1\right) \\
\bar{T}^{t, R} & =\frac{1}{M} \sum_{l=1}^{M} \sqrt{M}\left(-a \mathbb{I}_{(-\infty,-a)}\left(Z_{l}\right)+Z_{l} \mathbb{I}_{[-a, a]}\left(Z_{l}\right)+a \mathbb{I}_{(a, \infty)}\left(Z_{l}\right)\right) .
\end{aligned}
$$

We let $Z_{l} \stackrel{\text { i.i.d. }}{\sim} \mathrm{N}\left(\sqrt{M} \mu_{T}, 1\right)$. We use the Laplace mechanism based on the appropriate global sensitivity values to generate $\bar{T}^{t, \epsilon}$.

Each $T$ and $\bar{T}^{t, R}$ is generated independently. Generally, one would expect their values to be positively correlated when computed on some $\boldsymbol{D}$. However, generating them independently guarantees that $\mathbf{A 2}$ holds. Scenario I provides lower bounds for cases where the $t$-statistics are positively correlated, since the statistics should be more similar when positively correlated than when independent.

In Scenario II, we work with a subset of the March 2000 Current Population Survey (CPS) public use file comprising $n=49,436$ heads of households with non-negative incomes. This 
dataset was used by Reiter (2005) and Chen et al. (2016), among others. In order to use realistic predictor distributions, we set $\mathbf{X}_{D}$ to be an $n \times 25$ matrix of values derived from the CPS data. Its columns include age in years, age squared, education (16 levels), marital status (7 levels), and sex (2 levels). We generate multiple sets of the response variable $Y$ from linear regressions on $\mathbf{X}_{\boldsymbol{D}}$, each using a different, pre-specified set of $\boldsymbol{\beta}=\left(\beta_{0}, \ldots, \beta_{24}\right)^{\top}$, so as to control the importance of the regression coefficients. For $j=0, \ldots, 24$, let $\Sigma_{j, j}(\boldsymbol{D})$ be the $j$ th diagonal element of $\sigma^{2}\left(\mathbf{X}_{\boldsymbol{D}}^{\top} \mathbf{X}_{\boldsymbol{D}}\right)^{-1}$, where $\sigma=0.82$. This value of $\sigma$ corresponds to the residual standard error of a linear regression fitted using the logarithm of income-one of the variables in the CPS data-as the response variable and $\mathbf{X}_{\boldsymbol{D}}$ as predictors. To derive any one $\boldsymbol{\beta}=\left(\beta_{0}, \ldots, \beta_{24}\right)^{\top}$, we set each $\beta_{j}=\mu_{T} \sqrt{\Sigma_{j, j}(\boldsymbol{D})}$ for some specified number of standard deviations $\mu_{T}$ from zero. Using this $\boldsymbol{\beta}$, we simulate realizations of $T$ and $\bar{T}^{t, R}$ via the following steps.

i) For $i=1, \ldots, n$, generate $y_{i}$ from $\mathrm{N}\left(x_{i}^{\top} \boldsymbol{\beta}, \sigma^{2}\right)$, where $x_{i}$ denotes the $i$ th row-vector of $\mathbf{X}_{\boldsymbol{D}}$ and $\sigma=0.82$.

ii) Set $\boldsymbol{D}=\left\{\left(x_{i}, y_{i}\right)\right\}_{i=1}^{n}$, and generate a random partition $\mathcal{P}=\left\{\boldsymbol{D}_{l}\right\}_{l=1}^{M}$.

iii) Get a realization of $T=T(\boldsymbol{D})$ by computing the $t$-statistic of the $j$ th regression coefficient estimated from $D$.

iv) For $l=1, \ldots, M$, set $Z_{l}=T\left(D_{l}\right)$ as the $t$-statistic of the $j$ th regression coefficient obtained from the regression of $y$ on $\mathbf{X}_{\boldsymbol{D}_{l}}$. Get realizations of $\bar{T}^{t, R}$ from $[10)$.

We then add Laplace noise to generate $\bar{T}^{t, \epsilon}$.

For both scenarios, we let $\mu_{T} \in\{0,0.1, \ldots, 1,2, \ldots, 10\}$, let $M \in\{10,25,50,75,100\}$, let $a \in\{1, \ldots, 10\}$, and let $\epsilon \in\{0.5,1,2.5,5\}$. We generate simulations for all possible combinations of $\left(\mu_{T}, M, a, \epsilon\right)$. For each combination $\left(\mu_{T}, M, a, \epsilon\right)$, we generate 100,000 realizations of $\left(T, \bar{T}^{t, R}\right)$ for Scenario I and 1,000 realizations for Scenario II.

We evaluate the significance tests based on $\bar{T}^{t, \epsilon}$ by comparing the power at each value of $\mu_{T}$ to the power of the test based on $T$ at each corresponding value of $\mu_{T}$. We evaluate the 
properties of the differentially private sign measures by comparing how often one can infer the correct sign of each $\beta_{j}$ from the corresponding $T$ and $\bar{T}^{t, \epsilon}$. We also compute the probability that a user makes the same decision about the significance level or sign when using $T$ and $\bar{T}^{t, \epsilon}$; we call these matching probabilities.

\subsection{Assessing inferences about significance}

We study the significance properties of the $t$-statistics using the following quantities,

$$
\begin{aligned}
p_{0}(\mathrm{t}, \gamma) & =P\left\{|T|<\mathrm{t} \mid \mu_{T}=\gamma, \mathbf{X}_{\boldsymbol{D}}\right\} \\
p_{t, \epsilon}(\mathrm{t}, \gamma, M, a, \epsilon) & =P\left\{\left|\bar{T}^{t, \epsilon}\right|<\mathrm{t} \mid \mu_{T}=\gamma, M, a, \epsilon, \mathbf{X}_{\boldsymbol{D}}, \mathcal{R}\right\}
\end{aligned}
$$

As a slight abuse of notation, we condition these probabilities on $(\gamma, M, a, \epsilon)$ to highlight that they parametrize the probabilities.

For a given significance level $\alpha$ and type II error rate $\lambda_{0}$, let $r_{0}$ and $q_{0}$ be positive constants such that $p_{0}\left(r_{0}, 0\right)=1-\alpha$ and $p_{0}\left(r_{0}, q_{0}\right)=\lambda_{0}$. Notice that $r_{0}$ is the $(1-\alpha / 2)$ th quantile of the distribution of $T$ when $\mu_{T}=0$, i.e., $r_{0}$ is the critical value under the null hypothesis $H_{0}: \beta_{j}=0$ that ensures a confidence level of $1-\alpha$ for the test $\mathbb{T}=\mathbb{I}_{\left(-r_{0}, r_{0}\right)}(T)$. The value $q_{0}$ is the number of standard deviations from zero at which the test $\mathbb{T}$ reaches the desired Type II error $\lambda_{0}$, i.e., under $H_{1}: \beta_{j}=q_{0} \sqrt{\Sigma_{j, j}(\boldsymbol{D})}$, the power of this test is equal to $1-\lambda_{0}$.

Let $r$ and $\lambda(M, a, \epsilon)$ be positive constants such that $p_{t, \epsilon}(r, 0, M, a, \epsilon)=1-\alpha$ and $\lambda(M, a, \epsilon)=$ $p_{t, \epsilon}\left(r, q_{0}, M, a, \epsilon\right)$. For given values of $(M, a, \epsilon), r$ is the critical value under $H_{0}$ that ensures a confidence level of $1-\alpha$ for the test $\mathbb{T}_{t, \epsilon}=\mathbb{I}_{(-r, r)}\left(\bar{T}^{t, \epsilon}\right)$. The value $\lambda(M, a, \epsilon)$ corresponds to the type II error rate of $\mathbb{T}_{t, \epsilon}$ under $H_{1}: \beta_{j}=q_{0} \sqrt{\Sigma_{j, j}(\boldsymbol{D})}$.

To assess how similar the test $\mathbb{T}_{t, \epsilon}$ is to $\mathbb{T}$, we use the loss function,

$$
L_{t, \epsilon}^{s i g}(M, a, \epsilon)=\max \left(0, \lambda(M, a, \epsilon)-\lambda_{0}\right) .
$$


For a given significance level $\alpha$, we say there is zero loss of power from using $\mathbb{T}_{t, \epsilon}$ when the type II error rate of $\mathbb{T}_{t, \epsilon}$ at $q_{0}$ is less than $\lambda_{0}$. Otherwise, we record the corresponding loss of power, $\lambda(M, a, \epsilon)-\lambda_{0}$. For all results in this subsection, we set $\alpha=0.05$ and $\lambda_{0}=0.2$.

We begin by examining the values of $L_{t, \epsilon}^{s i g}(M, a, \epsilon)$ at the different combinations of $(M, a)$ when $\epsilon=\infty$, i.e., no noise is added to $\bar{T}^{t, \epsilon}$. To save space, we present these results in the supplementary material. In Scenario I, the power for $\mathbb{T}_{t, \epsilon}$ and $\mathbb{T}$ are almost identical for $a \geq 2$ for all values of $M$. This finding conforms with the theory in Section 4 . The results with the CPS data in Scenario II follow a similar pattern except for $M=100$. This is expected since large values of $M$ weaken the validity of $\mathbf{A 2}$.

We next consider $\epsilon<\infty$, as needed to satisfy differential privacy. Figure 3 displays values of $L_{t, \epsilon}^{s i g}(M, a, \epsilon)$ for $\epsilon \in\{0.5,1.0,2.5,5.0\}$. In Scenario I, $L_{t, \epsilon}^{s i g}(M, a, \epsilon)$ tends to be smaller when $M$ is large and $a$ is small, and largest when $M$ is small and $a$ is large. In other words, the discrepancy between the power of $\mathbb{T}_{t, \epsilon}$ and $\mathbb{T}$ is smaller when the global sensitivity is smallest. For values of $\epsilon>1$, there is at least one combination of $(M, a)$ for $\mathbb{T}_{t, \epsilon}$ that provides almost no loss in power. For $\epsilon=1$, with $\mathbb{T}_{t, \epsilon}$ we need $M \geq 50$ and $a \in\{1,2\}$ to experience only a small power loss. For $\epsilon=0.5$, with $\mathbb{T}_{t, \epsilon}$ we still can achieve only modest power losses by using $(M \geq 75, a=1)$. As expected, the power of $\mathbb{T}_{t, \epsilon}$ is strongly influenced by $\epsilon$. As $\epsilon$ gets smaller, so does the power. The reduction in power for small values of $\epsilon$ is the price to pay for strengthening the privacy guarantee. However, we emphasize that $\mathbb{T}_{t, \epsilon}$ is still a valid test when $\epsilon$ is small, in that it gives the correct type I error rates regardless of the value of $\epsilon$. Finally, the result patterns obtained under Scenario II generally match those obtained under Scenario I except for $M=100$. For this value of $M$, we notice a loss of power for some of the regression coefficients. We attribute this discrepancy to the fact that large values of $M$ weaken the validity of $\mathbf{A 2}$. 


\subsection{Assessing inferences about signs of coefficients}

Since the sign of $\mu_{T}$ and $\beta_{j}$ is the same, we restrict our analysis to the sign of $\mu_{T}$ only. Because the Laplace and Student- $t$ distributions are symmetric, we only consider the case where $\mu_{T} \geq 0$. We study the sign of the statistics using the following quantities,

$$
\begin{gathered}
s_{0}(\gamma)=P\left\{\operatorname{sign}(T)=\operatorname{sign}\left(\mu_{T}\right) \mid \mu_{T}=\gamma, \mathbf{X}_{\boldsymbol{D}}\right\} \\
s_{t, \epsilon}(\gamma, M, a, \epsilon)=P\left\{\operatorname{sign}\left(\bar{T}^{t, \epsilon}\right)=\operatorname{sign}\left(\mu_{T}\right) \mid \mu_{T}=\gamma, M, a, \epsilon, \mathbf{X}_{\boldsymbol{D}}, \mathcal{R}\right\} .
\end{gathered}
$$

These represent probabilities that the $t$-statistics have the same sign as $\beta_{j}$ when the value of $\beta_{j}$ is $\gamma$ standard deviations from zero. For a given probability $\alpha_{0}$, let $\mu_{0}$ be a positive constant such that $s_{0}\left(\mu_{0}\right)=\alpha_{0}$, i.e., $\mu_{0}$ is the number of standard deviations at which $T(\boldsymbol{D})$ has the same sign of $\beta_{j}$ with a probability equal to $\alpha_{0}$. To assess how similar $\alpha_{0}$ is to $s_{t, \epsilon}\left(\mu_{0}, M, a, \epsilon\right)$, we use the loss function

$$
L_{t, \epsilon}^{s g n}(M, a, \epsilon)=\max \left(0, \alpha_{0}-s_{t, \epsilon}\left(\mu_{0}, M, a, \epsilon\right)\right) .
$$

We say that $\bar{T}^{t, \epsilon}$ and $T(\boldsymbol{D})$ result in similar inferences about the sign of $\beta_{j}$ when $\alpha_{0}>s_{t, \epsilon}\left(\mu_{0}, M, a, \epsilon\right)$. For all results in this subsection, we set $\alpha_{0}=0.95$.

We again begin with the values of $L_{t, \epsilon}^{s g n}(M, a, \epsilon)$ for $\epsilon=\infty$; results are displayed in the supplementary material. Across scenarios, inferences about the sign of $\beta_{j}$ based on $\bar{T}^{t, \epsilon=\infty}$ are quite accurate for all combinations of $(M, a)$. Figure 4 displays results for $L_{t, \epsilon}^{s g n}(M, a, \epsilon)$ when $\epsilon \in\{0.5,1.0,2.5,5.0\}$. In general, when $\epsilon \leq 2.5, \bar{T}^{t, \epsilon}$ offers many combinations of $(M, a)$ that result in accurate inferences about the sign of $\beta_{j}$, especially when $a$ is small and $M$ is large. We also find combinations of $(M, a)$ that result in accurate inferences about the sign even when $\epsilon=0.5$, in particular when $M \geq 50$ and $a \in\{1,2\}$. When $\epsilon=5$, the results for $\bar{T}^{t, \epsilon}$ are practically indistinguishable for almost all combinations of $(M, a)$. These general findings hold for both scenarios, so that $\bar{T}^{t, \epsilon}$ provides a differentially private mechanism to release the sign of $\beta_{j}$ that seems robust against violations of $\mathbf{A 2}$. 


\subsection{Matching probabilities}

We define matching probabilities as the probability that $\operatorname{sign}(T)=\operatorname{sign}\left(\bar{T}^{t, \epsilon}\right)$ and the probability that $\mathbb{T}_{t, \epsilon}=\mathbb{T}$. We assess these probabilities using the quantities,

$$
\begin{aligned}
& m_{t, \epsilon}^{s i g}\left(\mu_{T}, \epsilon\right)=\min _{M, a} P\left\{\mathbb{T}_{t, \epsilon}=\mathbb{T} \mid \mu_{T}, M, a, \epsilon, \mathbf{X}_{\boldsymbol{D}}, \mathcal{R}\right\}, \\
& m_{t, \epsilon}^{s g n}\left(\mu_{T}, \epsilon\right)=\min _{M, a} P\left\{\operatorname{sign}(T)=\operatorname{sign}\left(\bar{T}^{t, \epsilon}\right) \mid \mu_{T}, M, a, \epsilon, \mathbf{X}_{\boldsymbol{D}}, \mathcal{R}\right\},
\end{aligned}
$$

where each minimum is over all possible combinations of $M \in\{10,25,50,75,100\}$ and $a \in\{1,2, \ldots, 10\}$. As we minimize over $(M, a)$, these metrics represent the worst case matching probabilities for these simulations. In the supplementary material, we present analogous figures showing the maximum values of the matching probabilities, which represent the best case matching probabilities for these simulations.

Figure 5 displays the values of $m_{t, \epsilon}^{s i g}\left(\mu_{T}, \epsilon\right)$ for different values of $\mu_{T}$ and $\epsilon$. The results in Scenario I and II follow similar patterns so we describe them simultaneously. Under the null hypothesis, i.e., $\mu_{T}=0$, values of $m_{t, \epsilon}^{s i g}$ are greater than 0.85 . When $\mu_{T}$ is large enough, values of $m_{t, \epsilon}^{s i g}$ are close to one. The value of $\mu_{T}$ at which $m_{t, \epsilon}^{s i g}$ is close to one is inversely related to the value of $\epsilon$. For example, when $\epsilon=5$ and $\mu_{T}>5$, then $m_{t, \epsilon}^{s i g} \approx 1$. However, when $\epsilon=0.5$, $m_{t, \epsilon}^{s i g}<0.5$ for all values of $\mu_{T}$. Values of $m_{t, \epsilon}^{s i g}$ tend to be small when $\mu_{T} \in\left[r_{0}, r\right]$, where $r_{0}$ and $r$ are the critical values associated with $\mathbb{T}$ and $\mathbb{T}_{t, \epsilon}$, respectively. Because $r$ increases as $\epsilon$ decreases, the range of values of $\mu_{T}$ where $m_{t, \epsilon}^{s i g}$ is small becomes wider as $\epsilon$ decreases.

Figure 5 also summarizes the results for $m_{t, \epsilon}^{s g n}\left(\mu_{T}, \epsilon\right)$. As expected, in both scenarios, increases in $\mu_{T}$ correspond to increases in the matching probability. The rate at which $m_{t, \epsilon}^{s g n}$ increases as a function of $\mu_{T}$ depends on $\epsilon$ : the larger the $\epsilon$, the faster the rate. In both scenarios, we observe a high matching probability $\left(m_{t, \epsilon}^{s g n}>0.9\right)$ when $\mu_{T} \geq 3$ and $\epsilon \geq 2.5$. When $\mu_{T}<3, m_{t, \epsilon}^{s g n}$ ranges from 0.5 to 0.9 for almost all $\epsilon$. It reaches a minimum value when $\mu_{T}=0$, regardless of the value of $\epsilon$; however, when $\mu_{T}=0$, matching the sign of $T$ and $\bar{T}^{t, \epsilon}$ arguably is 
not important for interpretations.

\section{Choosing $M$ and $a$ Without Additional Privacy Loss}

To use these differentially private test statistics, the data producer or, when permitted in a verification server, the analyst first fixes the desired privacy level $\epsilon$ and then must select values for $M$ and $a$. Here, we consider an analyst who does not get to choose $\epsilon$ but does get to choose $(M, a)$. Analysts who get to choose $\epsilon$, e.g., when allocating a total privacy budget across multiple queries, could repeat the approach described here with different values of $\epsilon$.

In this section, we present a three step approach for selecting values of $(M, a)$ that does not incur additional privacy loss. We illustrate these steps with a regression analysis of the CPS data, using the same $\mathbf{X}_{\boldsymbol{D}}$ as before and the reported values of household income on a logarithmic scale as the response variable. This regression fits reasonably well without obvious violations of the assumption of i.i.d. Gaussian errors. We present the methodology for $\bar{T}^{t, \epsilon}$ with $\epsilon=1.5$. The three-step approach also can be used for the sign.

Step 1: Fix an upper bound for $L_{t, \epsilon}^{s i g}(M, a, \epsilon)$. To begin, the user specifies an upper bound for $L_{t, \epsilon}^{s i g}(M, a, \epsilon)$ for their desired significance level $\alpha$ and type II error rate $\lambda_{0}$. By fixing $\alpha$ and $\lambda_{0}$, it is implied that there exists $q_{0}>0$ such that the power of $T$ equals $\left(1-\lambda_{0}\right)$ when $\beta_{j}$ is $q_{0}$ standard deviations from zero. Thus, the bound for $L_{t, \epsilon}^{s i g}(M, a, \epsilon)$ represents the loss of power that a user is willing to accept by using $\bar{T}^{t, \epsilon}$ instead of using $T$ when $\beta_{j}$ is $q_{0}$ standard deviations from zero. In our illustrative example, we set $\alpha=0.05$ and $\lambda_{0}=0.2$, and fix an upper bound of 0.1 .

Step 2: Simulate values of $L_{t, \epsilon}^{\text {sig }}(M, a, \epsilon)$ for choices of $M$ and a. The user can simulate values of $L_{t, \epsilon}^{s i g}(M, a, \epsilon)$ for different combinations of $(M, a)$ using the strategy described in Scenario I of Section 5 . As an example, Table 1 displays the values of $L_{t, \epsilon}^{s i g}(M, a, \epsilon)$ for different combinations of $(M, a)$. Of course, this table is not comprehensive; users can create different tables with different combinations of $(M, a)$. Importantly, we do not compute the entries in 


\begin{tabular}{l|ccccc} 
& \multicolumn{5}{|c}{$M$} \\
$a$ & 10 & 25 & 50 & 75 & 100 \\
\hline 1 & 0.13 & $\mathbf{0 . 0 5}$ & $\mathbf{0 . 0 2}$ & $\mathbf{0 . 0 1}$ & $\mathbf{0 . 0 1}$ \\
2 & 0.17 & $\mathbf{0 . 0 5}$ & $\mathbf{0 . 0 1}$ & $\mathbf{0 . 0 1}$ & $\mathbf{0 . 0 0}$ \\
3 & 0.32 & 0.11 & $\mathbf{0 . 0 4}$ & $\mathbf{0 . 0 2}$ & $\mathbf{0 . 0 1}$ \\
4 & 0.51 & 0.22 & $\mathbf{0 . 1 0}$ & $\mathbf{0 . 0 6}$ & $\mathbf{0 . 0 4}$ \\
5 & 0.65 & 0.34 & 0.16 & $\mathbf{0 . 1 0}$ & $\mathbf{0 . 0 7}$ \\
6 & 0.74 & 0.47 & 0.25 & 0.16 & 0.12 \\
7 & 0.79 & 0.58 & 0.34 & 0.22 & 0.16 \\
8 & 0.82 & 0.66 & 0.43 & 0.30 & 0.21 \\
9 & 0.84 & 0.72 & 0.51 & 0.37 & 0.27 \\
10 & 0.86 & 0.77 & 0.59 & 0.44 & 0.34
\end{tabular}

Table 1: Theoretical values of $L_{t, \epsilon}^{s g n}(M, a, 1.5)$ at different combinations of $(a, M), \alpha=0.05$, and $\lambda_{0}=0.2$. Values in bold satisfy the condition $L_{t, \epsilon}^{s g n}(M, a, 1.5)<0.10$.

Table 1 using the CPS data; otherwise, the results would leak information about the confidential data. When auxiliary data are available, such as synthetic data, analysts could base the tables off these auxiliary data using the strategy described in Scenario II of Section 5 .

Step 3: Choose $(M, a)$. The user considers all values of $(M, a)$ corresponding to values of $L_{t, \epsilon}^{s i g}(M, a, \epsilon)$ below the fixed upper bound. When no combination of $(M, a)$ satisfies this condition, the user has to sacrifice accuracy and increase their error tolerance. Once possible solutions exist, we recommend that the user choose the smallest value of $M$ from these solutions. Smaller values of $M$ result in larger sample sizes within the partitions, lessening the possibility that $\mathbf{A 1}$ or A2 are invalid. In our example, based on Table 1, the user should choose the smallest values of $M$ for which $L_{t, \epsilon}^{s i g}(M, a, \epsilon=1.5)$ is below 0.1 , which is $M=25$. The user then chooses the value of $a$ that minimizes $L_{t, \epsilon}^{s i g}(M=25, a, \epsilon=1.5)$. From Table 1, $L_{t, \epsilon}^{s i g}(M=25, a, \epsilon=1.5)$ reaches its minimum at 0.05 for $\mathbb{T}_{t, \epsilon}$ and $a \in\{1,2\}$. In theory, any of these values of $a$ should work. In this case, we recommend $a=2$ since this truncation level has the least effect on the approximation to $T(\boldsymbol{D})$.

We now illustrate this method of choosing $(M, a)$ on the CPS data. Using $(M=25, a=2)$, we compute the differentially private p-value and sign using Algorithms 1 and 2; results for all 
25 coefficients are displayed in the supplementary material. The p-values from the differentially private significance test and the test based on the confidential data $\boldsymbol{D}$ agree substantially, resulting in essentially the same conclusions about the significance for most of the regression coefficients. We observe discrepancies when the p-values based on $\boldsymbol{D}$ provide weak evidence against the null. Regarding the sign, the outputs from the differentially private algorithm agree with the observed signs for almost all coefficients, except some with large p-values. When pvalues for the test of $H_{0}: \beta_{j}=0$ are quite large, changes in sign arguably are inconsequential.

As an additional illustration, we repeat the model selection and estimation using $\epsilon=0.5$ and upper bound for $L_{t, \epsilon}^{s i g}(M, a, \epsilon)$ equal to 0.2 ; detailed results are in the supplementary material. The $(M, a)$ selection algorithm suggests that we set $(M=100, a=1)$. For estimating the signs, the differentially private algorithms continue to be effective: the privately-computed and observed signs systematically agree on all but three of the coefficients with significant $\mathrm{p}$-values. For p-values, we see different interpretations of the significance for 3 out of 25 coefficients. We conjecture why this occurs when discussing the results in the supplementary material. Of course, interpretations about the quality of the results for different $(M, a)$ and $\epsilon$ are data-specific, and we expect lower reductions in data quality for $\boldsymbol{D}$ with larger sample size.

\section{Concluding Remarks}

The methods described here allow data producers to provide $\epsilon$-differentially private answers to queries about the statistical significance and signs of coefficients in linear regression models. Further, the strategy from Section 6 provides users with a principled way to choose $(M, a)$. We expect that these methods can be applied to significance testing with other regression models. The key assumptions are $\mathbf{A 1}$ and $\mathbf{A 2}$, which are reasonable for many models and data settings.

The methods extend trivially to two contexts that are not discussed in previous sections. First, we can test general null hypotheses, $H_{0}: \beta_{j}=b$ with $b \in \mathbb{R}$. We simply define the $t$-statistic as $\left(\hat{\beta}_{j}-b\right) / \sqrt{\hat{\Sigma}_{j, j}}$, and apply the algorithms without modification. One application 
of this extension, which we leave to future investigation, is to set $b$ equal to the output of a privately computed regression coefficient $\tilde{\beta}_{j}$. The test statistic then could be interpreted as a

noisy, truncated estimate of the number of standard errors $\tilde{\beta}_{j}$ is from $\hat{\beta}_{j}$. Second, we can test the significance of the intercept, i.e., $H_{0}: \beta_{0}=b$. This is equivalent to a $\epsilon$-DP, one sample t-test for a single mean. Unlike other tests for means, this significance test does not presume known bounds on data values or population means. Nonetheless, it would be interesting to compare the power of the various tests for single means to see when each offers the best performance.

The algorithm described here provides results for one $\beta_{j}$ at a time. If analysts are given a finite privacy budget, they must spend part of that budget for each coefficient they wish to verify. Thus, a key area for research is to develop differentially private algorithms that allow queries for multiple test statistics without burning through the privacy budget too quickly.

\section{Supplementary Material}

The online supplementary materials include a graphical representation of the upper bound provided in Theorem 2, and the proof of this theorem. They also include additional plots from simulations in Section 5.1 and Section 5.2 when $\epsilon=\infty$, and in Section 5.3 when the maximum of the matching probabilities is taken over $(M, a)$ for fixed values of $\epsilon$. Finally, the include the p-values and signs for the coefficients in the two examples considered in Section 6.

\section{Acknowledgments}

This work is supported by grants from the National Science Foundation (ACI 1443014 and SES 1131897) and the Alfred P. Sloan Foundation (G-2-15-20166003). 


\section{References}

John Abowd, Martha Stinson, and Gary Benedetto. Final report to the social security administration on the SIPP/SSA/IRS public use file project. Technical report, 2006. U.S. Census Bureau Longitudinal Employer-Household Dynamics Program. Available at http: //hdl.handle.net/1813/43929.

Raef Bassily, Adam Smith, and Abhradeep Thakurta. Private empirical risk minimization: Efficient algorithms and tight error bounds. In Foundations of Computer Science (FOCS), 2014 IEEE 55th Annual Symposium on, pages 464-473. IEEE, 2014.

Rabi Bhattacharya, Lizhen Lin, and Victor Patrangenaru. Course in Mathematical Statistics and Large Sample Theory. Springer, 2016.

Zachary Campbell, Andrew Bray, Anna Ritz, and Adam Groce. Differentially private anova testing. In Data Intelligence and Security (ICDIS), 2018 1st International Conference on, pages 281-285. IEEE, 2018.

Kamalika Chaudhuri and Claire Monteleoni. Privacy-preserving logistic regression. In Advances in Neural Information Processing Systems, pages 289-296, 2009.

Kamalika Chaudhuri, Claire Monteleoni, and Anand D Sarwate. Differentially private empirical risk minimization. Journal of Machine Learning Research, 12(Mar):1069-1109, 2011.

Yan Chen, Ashwin Machanavajjhala, Jerome P Reiter, and Andrés F Barrientos. Differentially private regression diagnostics. In 2016 IEEE 16th International Conference on Data Mining (ICDM), pages 81-90, 2016.

Merce Crosas. The dataverse network: An open-source application for sharing, discovering and preserving data. D-lib Magazine, 17(1/2), 2011.

Vito D'Orazio, James Honaker, and Gary King. Differential privacy for social science inference. 2015. 
J. Drechsler. Synthetic Datasets for Statistical Disclosure Control. New York: Springer-Verlag, 2011.

Cynthia Dwork and Jing Lei. Differential privacy and robust statistics. In Proceedings of the forty-first annual ACM symposium on Theory of computing, pages 371-380. ACM, 2009.

Cynthia Dwork, Frank McSherry, Kobbi Nissim, and Adam Smith. Calibrating Noise to Sensitivity in Private Data Analysis, pages 265-284. Springer Berlin Heidelberg, 2006.

Marco Gaboardi, James Honaker, Gary King, Kobbi Nissim, Jonathan Ullman, and Salil P. Vadhan. PSI $(\Psi)$ : a private data sharing interface. arXiv preprint arXiv: 1609.04340, $2016 \mathrm{a}$.

Marco Gaboardi, Hyun Lim, Ryan Rogers, and Salil Vadhan. Differentially private chisquared hypothesis testing: Goodness of fit and independence testing. arXiv preprint arXiv: 1602.03090, 2016b.

S. Gomatam, A. F. Karr, J. P. Reiter, and A. P. Sanil. Data dissemination and disclosure limitation in a world without microdata: A risk-utility framework for remote access servers. Statistical Science, 20:163-177, 2005.

Antti Honkela, Mrinal Das, Onur Dikmen, and Samuel Kaski. Efficient differentially private learning improves drug sensitivity prediction. arXiv preprint arXiv:1606.02109, 2016.

Lei Jing, AnneSophie Charest, Aleksandra Slavkovic, Adam Smith, and Stephen Fienberg. Differentially private model selection with penalized and constrained likelihood. Journal of the Royal Statistical Society: Series A (Statistics in Society), 181(3):609-633, 2018.

Vishesh Karwa and Salil Vadhan. Finite sample differentially private confidence intervals. arXiv preprint arXiv:1711.03908, 2017.

Vishesh Karwa and Salil Vadhan. Finite Sample Differentially Private Confidence Intervals. In Anna R. Karlin, editor, 9th Innovations in Theoretical Computer Science Conference (ITCS 
2018), volume 94 of Leibniz International Proceedings in Informatics (LIPIcs), pages 44:144:9, Dagstuhl, Germany, 2018. Schloss Dagstuhl-Leibniz-Zentrum fuer Informatik.

Vishesh Karwa, Dan Kifer, and Aleksandra B Slavković. Private posterior distributions from variational approximations. arXiv preprint arXiv:1511.07896, 2015.

Daniel Kifer, Adam Smith, and Abhradeep Thakurta. Private convex empirical risk minimization and high-dimensional regression. Journal of Machine Learning Research, 1(41):3-1, 2012.

Gary King. An introduction to the dataverse network as an infrastructure for data sharing. Sociological Methods \& Research, 36(2):173-199, 2007.

Satkartar K Kinney, Jerome P Reiter, Arnold P Reznek, Javier Miranda, Ron S Jarmin, and John M Abowd. Towards unrestricted public use business microdata: The synthetic longitudinal business database. International Statistical Review, 79(3):362-384, 2011.

Ashwin Machanavajjhala, Daniel Kifer, John Abowd, Johannes Gehrke, and Lars Vilhuber. Privacy: Theory meets practice on the map. In Data Engineering, 2008. ICDE 2008. IEEE 24th International Conference on, pages 277-286. IEEE, 2008.

David McClure and Jerome P Reiter. Differential privacy and statistical disclosure risk measures: An investigation with binary synthetic data. Trans. Data Privacy, 5(3):535-552, 2012.

Arvind Narayanan and Vitaly Shmatikov. Robust de-anonymization of large sparse datasets. In Security and Privacy, 2008. SP 2008. IEEE Symposium on, pages 111-125. IEEE, 2008.

Kobbi Nissim, Sofya Raskhodnikova, and Adam Smith. Smooth sensitivity and sampling in private data analysis. In Proceedings of the thirty-ninth annual ACM symposium on Theory of computing, pages 75-84. ACM, 2007.

C. O'Keefe and J. Chipperfield. A summary of attack methods and protective measures for fully automated remote analysis systems. International Statistical Review, 81:426-455, 2013. 
Christine M. O'Keefe and Norm M. Good. Regression output from a remote analysis server. Data \& Knowledge Engineering, 68(11):1175-1186, 2009.

Marc Parry and Jon Chase. Harvard researchers accused of breaching students' privacy. Chronicle, 1:30, 2011.

Trivellore E Raghunathan, Jerome P Reiter, and Donald B Rubin. Multiple imputation for statistical disclosure limitation. Journal of official statistics, 19(1):1, 2003.

Jerome P Reiter. Model diagnostics for remote access regression servers. Statistics and Computing, 13(4):371-380, 2003.

Jerome P Reiter. Releasing multiply imputed, synthetic public use microdata: an illustration and empirical study. Journal of the Royal Statistical Society: Series A (Statistics in Society), 168(1):185-205, 2005.

Jerome P Reiter and Christine N Kohnen. Categorical data regression diagnostics for remote access servers. Journal of Statistical Computation and Simulation, 75(11):889-903, 2005.

Jerome P Reiter, Anna Oganian, and Alan F Karr. Verification servers: Enabling analysts to assess the quality of inferences from public use data. Computational Statistics \& Data Analysis, 53(4):1475-1482, 2009.

Donald B. Rubin. Discussion: Statistical disclosure limitation. Journal of Official Statistics, 9: 462-468, 1993.

Tamás Sarlós. Improved approximation algorithms for large matrices via random projections. In 2006 47th Annual IEEE Symposium on Foundations of Computer Science (FOCS'06), pages 143-152, 2006.

Or Sheffet. Differentially private ordinary least squares: $t$-values, confidence intervals and rejecting null-hypotheses. arXiv preprint arXiv:1507.02482, 2015. 
Eftychia Solea. Differentially Private Hypothesis Testing For Normal Random Variables. PhD thesis, The Pennsylvania State University, 2014.

Latanya Sweeney. Weaving technology and policy together to maintain confidentiality. The Journal of Law, Medicine \& Ethics, 25(2-3):98-110, 1997.

Latanya Sweeney. Matching known patients to health records in washington state data. Technical report, 2013. Data Privacy Lab, Harvard University.

Aad W Van der Vaart. Asymptotic statistics (Cambridge series in statistical and probabilistic mathematics). Cambridge University Press, 2000.

Duy Vu and Aleksandra Slavkovic. Differential privacy for clinical trial data: Preliminary evaluations. In Data Mining Workshops, 2009. ICDMW'09. IEEE International Conference on, pages 138-143. IEEE, 2009.

Yue Wang, Jaewoo Lee, and Daniel Kifer. Differentially private hypothesis testing, revisited. arXiv preprint arXiv:1511.03376, 2017.

Larry Wasserman and Shuheng Zhou. A statistical framework for differential privacy. Journal of the American Statistical Association, 105(489):375-389, 2010.

Xi Wu, Matthew Fredrikson, Wentao Wu, Somesh Jha, and Jeffrey F Naughton. Revisiting differentially private regression: Lessons from learning theory and their consequences. arXiv preprint arXiv:1512.06388, 2015.

Jun Zhang, Zhenjie Zhang, Xiaokui Xiao, Yin Yang, and Marianne Winslett. Functional mechanism: regression analysis under differential privacy. Proceedings of the VLDB Endowment, 5(11):1364-1375, 2012. 

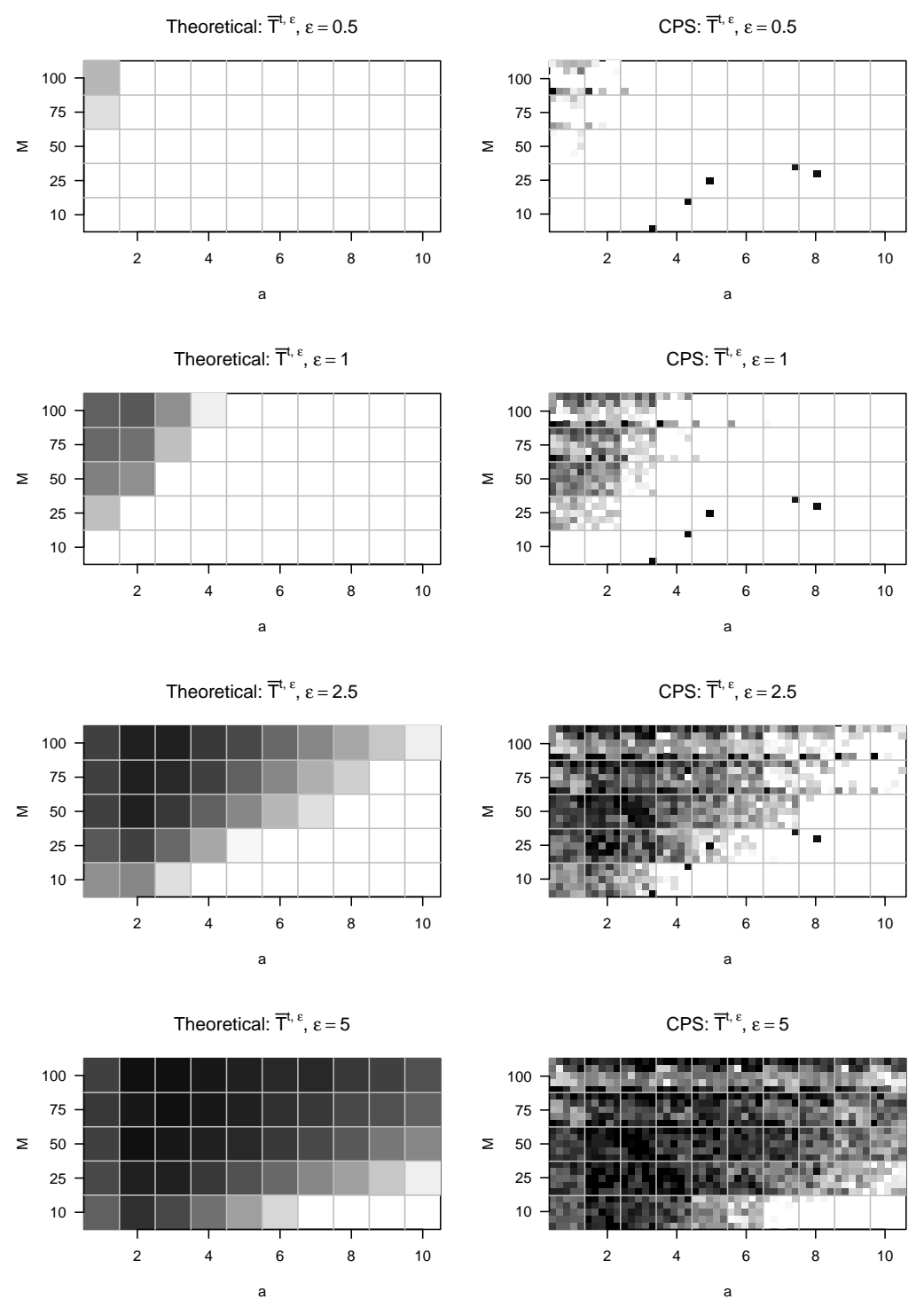

Figure 3: Values of $L_{t, \epsilon}^{s i g}(M, a, \epsilon)$ associated with $\bar{T}^{t, \epsilon}$ for significance at different combinations of $(M, a, \epsilon)$ with $\alpha=0.05$, and $\lambda_{0}=0.2$. Left and right panels show the results for Scenario I and II, respectively. For Scenario I, each cell represents the average of 100,000 runs at that $(M, a)$. For Scenario II, each $(M, a)$ cell comprises a $5 \times 5$ array of sub-cells representing the results for the 25 coefficients in the CPS regression. Individual sub-cell results are averages of 1,000 runs. Within any cell or sub-cell, black represents $L_{t, \epsilon}^{\text {sig }}(M, a, \epsilon)=0$ and white represents $L_{t, \epsilon}^{s i g}(M, a, \epsilon) \geq 0.25$, with lighter grays as the loss function approaches 0.25 . 

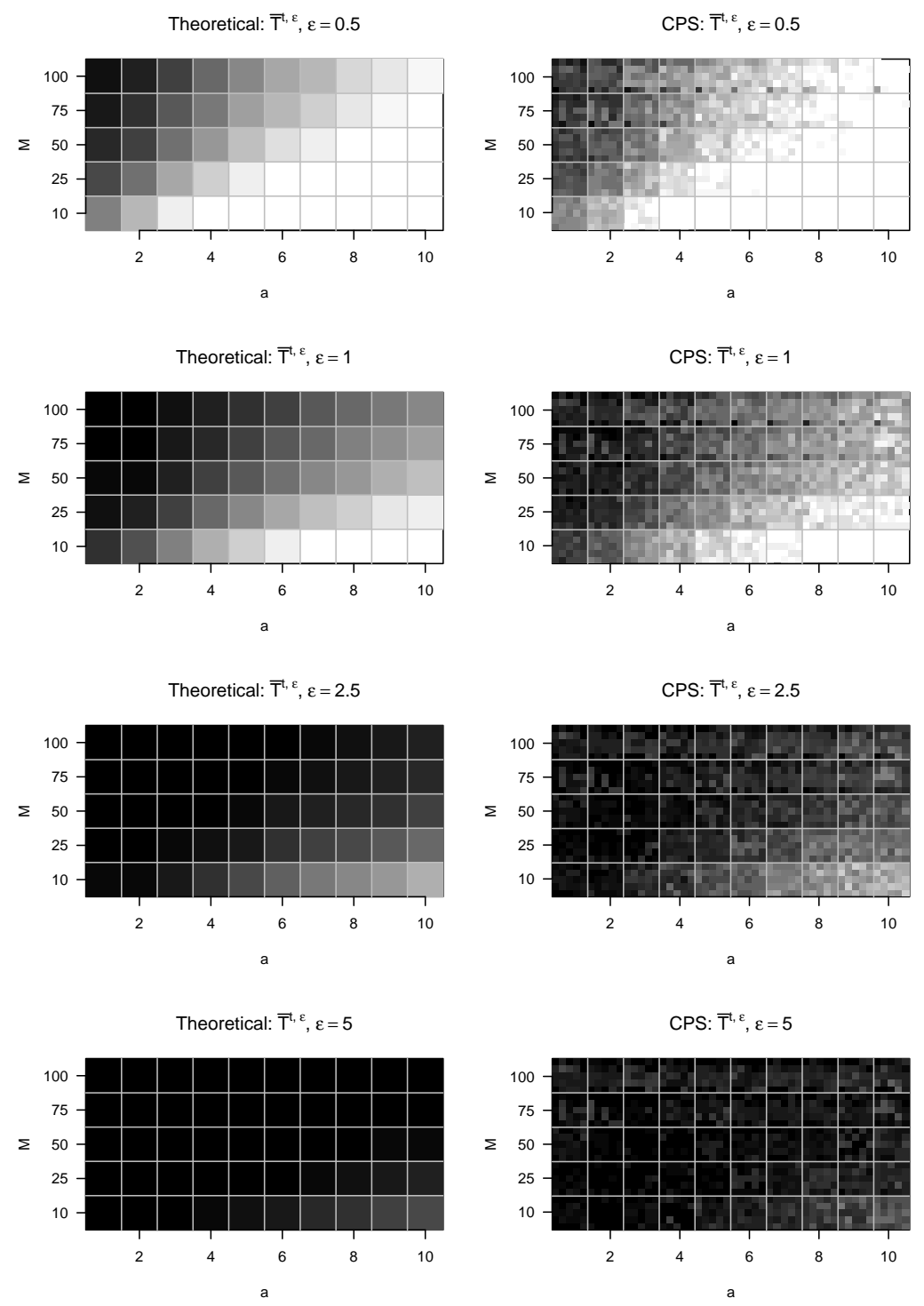

Figure 4: Values of $L_{t, \epsilon}^{s g n}(M, a, \epsilon)$ at different combinations of $(M, a, \epsilon)$ with $\alpha_{0}=0.95$. Left and right panels show the results for Scenario I and II, respectively. For Scenario I, each cell represents the average of 100,000 runs at that $(M, a)$. For Scenario II, each $(M, a)$ cell comprises a $5 \times 5$ array of sub-cells representing the results for the 25 coefficients in the CPS regression. Individual sub-cell results are averages of 1,000 runs. Within any cell or sub-cell, black represents $L_{t, \epsilon}^{s g n}(M, a, \epsilon)=0$ and white represents $L_{t, \epsilon}^{s g n}(M, a, \epsilon) \geq 0.25$, with lighter grays as the loss function approaches 0.25 . 

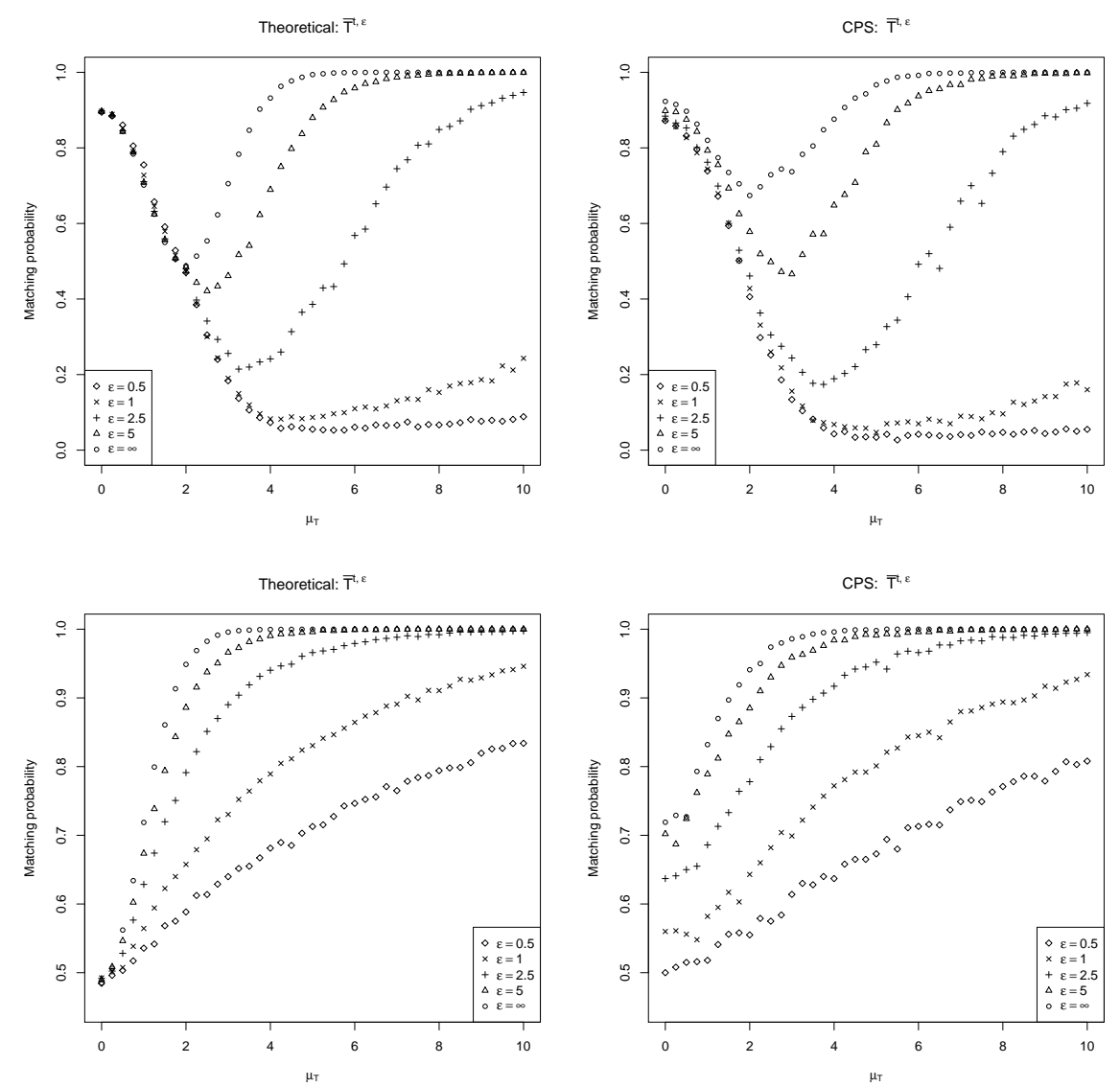

Figure 5: Values of $m_{t, \epsilon}^{s i g}\left(\mu_{T}, \epsilon\right)$ and $m_{t, \epsilon}^{s g n}\left(\mu_{T}, \epsilon\right)$ at different combinations of $\mu_{T}$ and $\epsilon$ with $\alpha=0.05$. Left and right panels show Scenario I and II, respectively. Top and bottom panels show the results for $m_{t, \epsilon}^{s i g}\left(\mu_{T}, \epsilon\right)$ and $m_{t, \epsilon}^{s g n}\left(\mu_{T}, \epsilon\right)$, respectively. For Scenario II, each point presents $\mu_{T}$ and the minimum value of $m_{k}^{s i g}\left(\mu_{T}, \epsilon\right)$ taken over the 25 regression coefficients. 